\title{
Jacobi forms over complex quadratic fields via the cubic Casimir operators
}

\author{
Kathrin Bringmann, Charles H. Conley and Olav K. Richter*
}

We dedicate this article to Harold Stark on the occasion of his $70^{\text {th }}$ birthday

\begin{abstract}
We prove that the center of the algebra of differential operators invariant under the action of the Jacobi group over a complex quadratic field is generated by two cubic Casimir operators, which we compute explicitly. In the spirit of Borel, we consider Jacobi forms over complex quadratic fields that are also eigenfunctions of these Casimir operators, a new approach in the complex case. Theta functions and Eisenstein series provide standard examples. In addition, we introduce an analog of Kohnen's plus space for modular forms of half-integral weight over $K=\mathbb{Q}(i)$, and provide a lift from it to the space of Jacobi forms over $K=\mathbb{Q}(i)$.
\end{abstract}

Mathematics Subject Classification (2010). Primary 11F50; Secondary 43A85.

Keywords. Complex quadratic fields: Jacobi forms, Kohnen's plus space, invariant differential operators.

\section{Introduction}

In 1985, Eichler and Zagier [7] systematically developed a theory of (holomorphic) Jacobi forms. That theory has since grown enormously, establishing deep connections to different types of automorphic forms (for example, see Skoruppa and Zagier [23] and Skoruppa [21]) and many other areas of mathematics and physics, such as the theory of Heegner points (see Gross, Kohnen, and Zagier [10]), the theory of elliptic genera (see Zagier [24]), string theory (for example, see Cardy [5]), and more recently, mock theta functions (see Zwegers [26]).

The classical Jacobi forms in [7] are holomorphic functions. More generally, the Maass-Jacobi forms over the rationals in Berndt and Schmidt [1] and Pitale [17] are eigenfunctions of differential operators invariant under the action of the real Jacobi group. Automorphic forms over complex quadratic fields are clearly not

\footnotetext{
* The first author was partially supported by NSF grant DMS-0757907. Parts of this paper were written while the first author was in residence at the Max Planck Institute for Mathematics in Bonn and the third author was in residence at the RWTH Aachen and also at the Max Planck Institute in Bonn. Both authors are grateful for the hospitality. The third author thanks Aloys Krieg in particular for providing a stimulating research environment.
} 
holomorphic functions, and so one needs an analyticity condition to take the place of the Cauchy-Riemann differential equations. Skogman [20] and Richter and Skogman [18] use Jacobi theta functions to construct prototypes of Jacobi forms over complex quadratic fields and arbitrary number fields, respectively, but they do not discuss any analyticity condition for Jacobi forms. In [4] we give such a condition and suggest a definition of Jacobi forms over complex quadratic fields. More precisely, we consider Jacobi forms over complex quadratic fields that are also eigenfunctions of a second order differential operator invariant under the action of the complex Jacobi group. In addition, we present explicit examples showing that the space of such forms is nonempty. Nevertheless, the definition in [4] has drawbacks. For one thing, all examples of such Jacobi forms constructed there have eigenvalue zero. In addition, it seems that the approach in [4] does not give any connections to other types of automorphic forms. This is somewhat unsatisfactory, since a theory of Jacobi forms should yield a fruitful interplay between different types of automorphic forms.

In this paper we suggest a new approach to Jacobi forms that is in the spirit of Borel's notion of an automorphic form for a reductive group [2]. The center of the universal enveloping algebra of the complex Jacobi group is generated by two conjugate linear elements and two conjugate cubic elements, which we call the Casimir elements. The linear elements act on Jacobi forms by scalars, and the operators by which the Casimir elements act generate the center of the algebra of invariant differential operators. We consider (see Definition 2) Jacobi forms over complex quadratic fields that are eigenfunctions of the cubic Casimir operators. The Eisenstein series in [4] and special cases of the theta functions in [20] and [18] are concrete examples, and in contrast to [4], the space of Jacobi forms here yields a truly continuous spectrum. Furthermore, we introduce an analog of Kohnen's plus space for modular forms of half-integral weight over $K=\mathbb{Q}(i)$. We proceed as in Kohnen [14] to construct a lift from said analog to the space of Jacobi forms over $K=\mathbb{Q}(i)$, which gives rise to new examples of Jacobi forms over $K=\mathbb{Q}(i)$. Our work may be viewed as the first step toward a rigorous theory of Jacobi forms over complex quadratic fields. It will be interesting to see if such Jacobi forms find important applications in the same way that classical Jacobi forms do.

This paper is organized as follows. We begin Section 2 with the definition of the Jacobi group of any subdomain of $\mathbb{C}$, and the $\left.\right|_{k, m}$-slash action of the complex Jacobi group. We then state the main result of Section 6, the description of the center of the algebra of $\left.\right|_{k, m}$-invariant operators, and use it to define Jacobi forms over complex quadratic fields.

Section 3 is the heart of the number theoretic part of the paper. It begins with the determination of the transformation laws of Jacobi theta functions. These theta functions are important tools in the proof of the main result of the section, which constructs new examples of Jacobi forms over $K=\mathbb{Q}(i)$ by lifting modular forms of half-integral weight (in the analog over $K=\mathbb{Q}(i)$ of Kohnen's plus space) via a theta decomposition. 
Sections 4-6 comprise the Lie theoretic part of the paper. In Section 4 we compute the generators and relations of the algebra of invariant differential operators on any homogeneous $G$-vector bundle in terms of $G$ 's universal enveloping algebra, $G$ being an arbitrary Lie group. The case that the stabilizer subgroup is connected and acts reductively on $g$ was treated by Helgason [11], and in fact this case is sufficient for Sections 5 and 6. We have included Section 4 for completeness and in order to give a slight generalization of Helgason's results.

The $\left.\right|_{k, m}$-slash action of the real Jacobi group was introduced in [7], and generators for its algebra of invariant differential operators were computed in [1]. In Section 5 we apply [11] to compute the relations in this algebra. We find that its center is the image of the center of the universal enveloping algebra of the Jacobi group, and is generated by a single cubic operator. This operator was used in [1] (page 82) to define Maass-Jacobi forms over the rationals, and such forms were studied in [17].

In Section 6 we apply [11] to the $\left.\right|_{k, m}$-slash action of the complex Jacobi group. Here the center of the algebra of $\left.\right|_{k, m}$-invariant operators is a polynomial algebra on two cubic operators, and it is again the image of the center of the universal enveloping algebra.

\section{Jacobi forms and the Jacobi group}

2.1. The Jacobi group. We follow the notation of [1], [4], and [7]. For any subdomain $D$ of $\mathbb{C}$, the centerless Jacobi group of $D$ is

$$
\bar{G}^{J}(D):=\mathrm{SL}_{2}(D) \ltimes D^{2} .
$$

We write elements of $D^{2}$ as row vectors $X$ and define the group law of $\bar{G}^{J}(D)$ via the right action of $\mathrm{SL}_{2}(D):(M, X)\left(M^{\prime}, X^{\prime}\right):=\left(M M^{\prime}, X M^{\prime}+X^{\prime}\right)$.

Let $D^{2} \tilde{x} D$ be the Heisenberg group of $D$, with group law

$$
(X, \kappa)\left(X^{\prime}, \kappa^{\prime}\right):=\left(X+X^{\prime}, \operatorname{det}\left(\begin{array}{l}
X \\
X^{\prime}
\end{array}\right)+\kappa+\kappa^{\prime}\right) .
$$

The Jacobi group of $D$ is $G^{J}(D):=\mathrm{SL}_{2}(D) \ltimes\left(D^{2} \tilde{\times} D\right)$, with group law

$$
(M, X, \kappa)\left(M^{\prime}, X^{\prime}, \kappa^{\prime}\right):=\left(M M^{\prime}, X M^{\prime}+X^{\prime}, \operatorname{det}\left(\begin{array}{c}
X M^{\prime} \\
X^{\prime}
\end{array}\right)+\kappa+\kappa^{\prime}\right) .
$$

Note that $Z^{J}(D):=\{I\} \times\{0\} \times D$ is the center of $G^{J}(D)$, and the projection $(M, X, \kappa) \mapsto(M, X)$ defines an isomorphism from $G^{J}(D) / Z^{J}(D)$ to $\bar{G}^{J}(D)$. It will be useful to define subgroups $K^{J}(D)$ of $G^{J}(D)$ and $\bar{K}^{J}(D)$ of $\bar{G}^{J}(D)$ by

$$
K^{J}(D):=\left(\operatorname{SU}_{2} \cap \mathrm{SL}_{2}(D)\right) \times\{0\} \times D, \quad \bar{K}^{J}(D):=\left(\operatorname{SU}_{2} \cap \mathrm{SL}_{2}(D)\right) \times\{0\} .
$$

Write $\mathcal{Q}$ for the ring of quaternions and $\mathbb{H}_{\mathcal{Q}}$ for the quaternionic upper half space:

$$
\begin{aligned}
\mathcal{Q} & :=\left\{u+v \mathbf{k} \mid u, v \in \mathbb{C}, \mathbf{k}^{2}=-1, a \mathbf{k}=\mathbf{k} \bar{a} \text { for all } a \in \mathbb{C}\right\}, \\
\mathbb{H}_{\mathcal{Q}} & :=\left\{x+y \mathbf{k} \in \mathcal{Q} \mid x \in \mathbb{C}, y \in \mathbb{R}^{+}\right\} .
\end{aligned}
$$


We denote elements of $\mathbb{H}_{\mathcal{Q}}$ by $\tau=x+y \mathbf{k}$ and elements of $\mathcal{Q}$ by $z=u+v \mathbf{k}$. Define

$$
\bar{z}:=\bar{u}-v \mathbf{k}, \quad \tilde{z}:=u+\bar{v} \mathbf{k}, \quad \mathcal{N}(z):=z \bar{z}=|u|^{2}+|v|^{2}
$$

(the usual quaternion norm). Also, set

$$
\|u+v \mathbf{k}\|_{\mathbb{C}}:=u+i v, \quad\|u+v \mathbf{k}\|_{\overline{\mathbb{C}}}:=\bar{u}+i \bar{v}, \quad \mathcal{T}_{\mathcal{R}}[z]:=\|z\|_{\mathbb{C}}+\|z\|_{\overline{\mathbb{C}}} .
$$

The following lemma is elementary; a conceptual proof may be found in [4]. For the rest of Section 2, set

$$
M:=\left(\begin{array}{cc}
\alpha & \beta \\
\gamma & \delta
\end{array}\right), \quad X:=(\lambda, \mu)
$$

Lemma 2.1. There is a transitive action of $\mathrm{SL}_{2}(\mathbb{C})$ on $\mathbb{H}_{\mathcal{Q}}$ defined by

$$
M \circ \tau:=(\alpha \tau+\beta)(\gamma \tau+\delta)^{-1}=(\tau \gamma+\delta)^{-1}(\tau \alpha+\beta),
$$

under which the stabilizer of $\mathbf{k}$ is $\mathrm{SU}_{2}$.

There is a transitive action of $G^{J}(\mathbb{C})$ on $\mathbb{H}_{\mathcal{Q}} \times \mathbb{Q}$ defined by

$$
(M, X, \kappa) \circ(\tau, z):=\left(M \circ \tau,(\tau \gamma+\delta)^{-1}(\tau \lambda+\mu+z)\right),
$$

under which the stabilizer of $(\mathbf{k}, 0)$ is $K^{J}(\mathbb{C})$. Division by $Z^{J}(\mathbb{C})$ yields a transitive action of $\bar{G}^{J}(\mathbb{C})$ on $\mathbb{H}_{\mathcal{Q}} \times \mathcal{Q}$, such that the stabilizer of $(\mathbf{k}, 0)$ is $\bar{K}^{J}(\mathbb{C})$.

We now define the $\left.\right|_{k, m}$-slash action of $G^{J}(\mathbb{C})$ on $C^{\infty}\left(\mathbb{H}_{\mathcal{Q}} \times \mathcal{Q}\right)$. Define a function $a: G^{J}(\mathbb{C}) \times\left(\mathbb{H}_{\mathcal{Q}} \times \mathbb{Q}\right) \rightarrow \mathbb{C}$ by

$a((M, X, \kappa),(\tau, z)):=\kappa+\lambda \mu+2 \lambda z+\lambda \tau \lambda-(\tilde{z}+\lambda \tau+\mu)(\gamma \tau+\delta)^{-1} \gamma(z+\tau \lambda+\mu)$.

For $k \in \mathbb{C}, l \in \mathbb{N}$, and $m \in \mathbb{C}^{l}$, define $\alpha_{k, m}: G^{J}(\mathbb{C}) \times\left(\mathbb{H}_{\mathcal{Q}} \times \mathcal{Q}\right) \rightarrow \mathbb{C}^{\times}$by

$$
\alpha_{k, m}((M, X, \kappa),(\tau, z)):=\mathcal{N}^{-k}(\gamma \tau+\delta) \exp \left\{2 \pi i \mathcal{T}_{\mathcal{R}}\left[{ }^{t} m a((M, X, \kappa),(\tau, z)) m\right]\right\} .
$$

Proposition 2.2. For all $k \in \mathbb{C}$ and $m \in \mathbb{C}^{l}$, the following equation defines a slash action $\left.\right|_{k, m}$ of $G^{J}(\mathbb{C})$ on functions $f: \mathbb{H}_{\mathcal{Q}} \times \mathcal{Q} \rightarrow \mathbb{C}$ :

$$
\left(\left.f\right|_{k, m}(M, X, \kappa)\right)(\tau, z):=\alpha_{k, m}((M, X, \kappa),(\tau, z)) f((M, X, \kappa) \circ(\tau, z)) .
$$

Multiplication by $y^{-k^{\prime}}$ is an intertwining map from $\left.\right|_{k, m}$ to $\left.\right|_{k+k^{\prime}, m}$ :

$$
\left.\left(y^{-k^{\prime}} f\right)\right|_{k+k^{\prime}, m}(M, X, \kappa)=\left.y^{-k^{\prime}}(f)\right|_{k, m}(M, X, \kappa) .
$$


This proposition amounts to the fact that $\alpha_{k, m}$ is a cocycle with respect to the action of $G^{J}(\mathbb{C})$ on $\mathbb{H}_{\mathcal{Q}} \times \mathcal{Q}$, such that $\alpha_{k+k^{\prime}, m} / \alpha_{k, m}$ is the coboundary $\partial y^{-k^{\prime}}$; see Section 4.2. It is proven in Section 5.3 of [4].

We now state the main results of the Lie-theoretic part of the paper: the description of the center of the algebra of differential operators on $\mathbb{H}_{\mathcal{Q}} \times \mathcal{Q}$ invariant with respect to the action $\left.\right|_{k, m}$ of $G^{J}(\mathbb{C})$, and the description of the action of the center of the universal enveloping algebra of $G^{J}(\mathbb{C})$ under $\left.\right|_{k, m}$. The two are the same and motivate the definition of Jacobi forms given in Section 2.2.

We will see in Section 6 that if we regard $G^{J}(\mathbb{C})$ as a complex group, then the center of its universal enveloping algebra is a polynomial ring on two generators, one linear and one cubic. It follows that if we forget its complex structure and regard it as a real group, the center of its universal enveloping algebra is a polynomial ring on four generators, two conjugate linear elements and two conjugate cubic elements. Under the real action $\left.\right|_{k, m}$, the cubic elements act by third order differential operators which we denote by $\ell_{k, m}^{ \pm}$, and the linear elements act by scalars. Thus $\ell_{k, m}^{ \pm}$generate the $\left.\right|_{k, m}$-action of the universal enveloping algebra of $G^{J}(\mathbb{C})$.

We will define $e_{k, m}^{+}$in terms of the following operators:

$$
\begin{aligned}
& Z_{k, m}^{+}:=-2 \pi i^{t} m m, \\
& e_{k, m}^{+}:=-\partial_{u}, \\
& E_{k, m}^{+}:=-\partial_{x}, \\
& H_{k, m}^{+}:=-\left(2 x \partial_{x}+y \partial_{y}+u \partial_{u}+v \partial_{v}+k\right), \\
& f_{k, m}^{+}:=-\left(x \partial_{u}+y \partial_{\bar{v}}\right)+4 \pi i^{t} m(m u+i \bar{m} v), \\
& F_{k, m}^{+}:=-x H_{k, m}^{+}-\left(x^{2} \partial_{x}+y^{2} \partial_{\bar{x}}\right)+y\left(u \partial_{\bar{v}}-v \partial_{\bar{u}}\right) \\
& \quad+2 \pi i^{t}(m u+i \bar{m} v)(m u+i \bar{m} v) .
\end{aligned}
$$

In order to define $e_{k, m}^{-}$, write $B$ for any of $Z, e, E, H, f, F$ and let

$$
B_{k, m}^{-}:=\overline{B_{k, i m}^{+}}
$$

Remark. It is useful to note that $B_{k, m}^{-}$and hence also $\bigodot_{k, m}^{-}$may be obtained from $B_{k, m}^{+}$and $\ell_{k, m}^{+}$by replacing $x, u, v, k$, and $m$ by their conjugates.

Definition 1. The Casmir operators of the action $\left.\right|_{k, m}$ are

$$
\begin{aligned}
e_{k, m}^{ \pm}:=\frac{i}{\pi}\left(\left(f_{k, m}^{ \pm}\right)^{2} E_{k, m}^{ \pm}-F_{k, m}^{ \pm}\left(e_{k, m}^{ \pm}\right)^{2}-\left(H_{k, m}^{ \pm}+2\right) f_{k, m}^{ \pm} e_{k, m}^{ \pm}\right. \\
\left.+\left(H_{k, m}^{ \pm}+1\right)\left(H_{k, m}^{ \pm}+2\right) Z_{k, m}^{ \pm}+4 F_{k, m}^{ \pm} E_{k, m}^{ \pm} Z_{k, m}^{ \pm}\right) .
\end{aligned}
$$


Theorem 2.3. The third order differential operators $\bigcup_{k, m}^{ \pm}$on $\mathbb{H}_{\mathcal{Q}} \times \mathcal{Q}$ are invariant with respect to the action $\left.\right|_{k, m}$ of $G^{J}(\mathbb{C})$. They generate a polynomial algebra in two variables which is both the center of the algebra of all $\left.\right|_{k, m}$-invariant differential operators and the image of the center of the universal enveloping algebra of $G^{J}(\mathbb{C})$ under $\left.\right|_{k, m}$.

The operators $\mathcal{C}_{k, m}^{ \pm}$are the conjugates of $\bigcup_{0, m}^{ \pm}$by $y^{-k}$ :

$$
\varphi_{k, m}^{ \pm}\left(y^{-k} f(\tau, z)\right)=y^{-k} \varphi_{0, m}^{ \pm} f(\tau, z) .
$$

We now discuss the following slash action of $\mathrm{SL}_{2}(\mathbb{C})$ on $\mathbb{H}_{Q}$ :

$$
\left(\left.f\right|_{k} M\right)(\tau):=\mathcal{N}^{-k}(\gamma \tau+\delta) f(M \circ \tau)
$$

(see (1)). It is easy to check directly that this is an action for all $k \in \mathbb{C}$, and that multiplication by $y^{-k^{\prime}}$ is an intertwining map from $\left.\right|_{k}$ to $\left.\right|_{k+k^{\prime}}$. This also follows from Proposition 2.2 and the observation that $\left.\right|_{k}$ is the restriction of $\left.\right|_{k, 0}$ to an action of $\mathrm{SL}_{2}(\mathbb{C})$ on functions constant in $z$.

Define a second order operator $\Delta_{k}$ on $\mathbb{H}_{\mathcal{Q}}$ by

$$
\Delta_{k}:=y^{2}\left(4 \partial_{x} \partial_{\bar{x}}+\partial_{y}^{2}\right)+(2 k-1) y \partial_{y} .
$$

Note that $\Delta_{0}$ is the usual Laplace-Beltrami operator, which is well known to generate the algebra of $\left.\right|_{0}$-invariant differential operators, and $\Delta_{k}+k^{2}-2 k$ is its conjugate by $y^{-k}$ :

$$
\Delta_{k}\left(y^{-k} f\right)=y^{-k}\left(\Delta_{0}(f)-\left(k^{2}-2 k\right) f\right) .
$$

It follows that $\Delta_{k}$ generates the algebra of $\left.\right|_{k}$-invariant operators.

Clearly restriction to functions constant in $z$ defines an algebra map from $\left.\right|_{k, 0^{-}}$ invariant operators to $\left.\right|_{k}$-invariant operators. Since $e_{k, 0}^{ \pm}$and $f_{k, 0}^{ \pm}$annihilate functions constant in $z$ and $Z_{k, 0}^{ \pm}=0$, this algebra map sends $\ell_{k, 0}^{ \pm}$to 0 . We remark that it sends the operators $D_{k, 0}^{\tau}$ and $D_{k, 0}^{z}$ in (4) of [4] to $\Delta_{k}$ and 0, respectively. (Let us take this opportunity to correct an error in Proposition 5.7 of [4]: the first displayed equation should read $D_{k, m}^{\tau}=\operatorname{IDO}_{\alpha_{k, m}}\left(Q_{\tau} / 4\right)-k^{2}+2 k$.)

2.2. Jacobi forms. Fix a complex quadratic field $K$ with discriminant $d_{K}$. Algebraic and complex conjugation are identical on $K$ and are denoted by $\alpha \mapsto \bar{\alpha}$. Let $\delta_{K}$ be the different of $K$ and let $\mathcal{O}_{K}$ be the ring of integers of $K$. The discrete Jacobi group $\Gamma^{J}(K)$ over $K$ is the centerless Jacobi group of $\mathcal{O}_{K}$ :

$$
\Gamma^{J}(K):=\bar{G}^{J}\left(\mathcal{O}_{K}\right)=\operatorname{SL}_{2}\left(\mathcal{O}_{K}\right) \ltimes \mathcal{O}_{K}^{2} .
$$

Proposition 2.4. The restriction to $G^{J}\left(\mathcal{O}_{K}\right)$ of the action $\left.\right|_{k, m}$ defined in Proposition 2.2 factors through to an action of the quotient $\Gamma^{J}(K)$ if and only if ${ }^{t} m m \in \mathfrak{D}_{K}^{-1}$. In particular, this holds for $K=Q(i)$ if and only if $2^{t} \mathrm{~mm} \in \mathbb{Z}[i]$. 
Writing $z^{*}$ for $(z+\tau \lambda+\mu)$, the resulting action of $\Gamma^{J}(K)$ is given by

$$
\begin{aligned}
& \left(\left.f\right|_{k, m}\left[\left(\begin{array}{ll}
\alpha & \beta \\
\gamma & \delta
\end{array}\right),(\lambda, \mu)\right]\right)(\tau, z):=f\left(\left[\left(\begin{array}{ll}
\alpha & \beta \\
\gamma & \delta
\end{array}\right),(\lambda, \mu)\right] \circ(\tau, z)\right) \\
& \quad \times \mathcal{N}(\gamma \tau+\delta)^{-k} \exp \left\{2 \pi i \mathcal{T}_{\mathcal{R}}\left[{ }^{t} m\left(-\widetilde{z^{*}}(\gamma \tau+\delta)^{-1} \gamma z^{*}+\lambda \tau \lambda+2 \lambda z\right) m\right]\right\} .
\end{aligned}
$$

Proof. This proposition is a sharpening of Lemma 5.6 of [4]. Equation (4) defines an action of $\Gamma^{J}(K)$ if and only if $\alpha_{k, m}$ is 1 on $Z^{J}\left(\mathcal{O}_{K}\right)$, which in turn occurs if and only if the factor $\exp \left\{2 \pi i \mathcal{T}_{\mathcal{R}}\left[{ }^{t} m m \kappa\right]\right\}$ of $\alpha_{k, m}$ arising from the summand $\kappa$ in (3) is 1 for all $\kappa \in \mathcal{O}_{K}$. In this case (4) reduces to (9) because the summand $\lambda \mu$ in (3) also contributes nothing. Since $\mathcal{T}_{\mathcal{R}}\left[{ }^{t} m m \mathcal{O}_{K}\right] \subseteq \mathbb{Z}$ if and only if ${ }^{t} m m \in \delta_{K}^{-1}=d_{K}^{-1 / 2} \mathcal{O}_{K}$, the result follows.

The definition of a (non-holomorphic) automorphic form requires an analyticity condition to take the place of the Cauchy-Riemann differential equations for holomorphic functions. On reductive groups one usually uses the action of the center of the universal enveloping algebra to give such a condition (see Borel [2]). The Jacobi group is not reductive, but nevertheless, in light of Theorem 2.3, the following definition of Jacobi forms fits into this framework. Moreover, it suggests a fruitful theory.

Definition 2. Let $k$ be in $\mathbb{N}$, and let $m$ be an element of $\mathbb{C}^{l}$ such that ${ }^{t} m m \in \delta_{K}^{-1}$ as in Proposition 2.4. A real-analytic function $f: \mathbb{H}_{\mathcal{Q}} \times \mathcal{Q} \rightarrow \mathbb{C}$ is a Jacobi form over $K$ of weight $k$, index vector $m$, and eigenvalues $\lambda^{+}, \lambda^{-} \in \mathbb{C}$ if

(i) $\left(\left.f\right|_{k, m} A\right)(\tau, z)=f(\tau, z)$ for all $A \in \Gamma^{J}(K)$,

(ii) $e_{k, m}^{+} f=\lambda^{+} f$ and $e_{k, m}^{-} f=\lambda^{-} f$,

(iii) $f$ is of moderate growth.

We denote the vector space of such Jacobi forms by $J_{k, m, \lambda+, \lambda-}$. In addition, we say that $\phi \in J_{k, m, \lambda+, \lambda-}$ is a cusp form if

$$
\phi(\tau, z)=O\left(\exp \{-b y\} \exp \left\{2 \pi|v|^{2} / y\right\}\right) \quad \text { as } y \rightarrow \infty \text { for some } b>0,
$$

which is analogous to the characterization of the Jacobi cusp forms in Skoruppa [22].

Explicit examples are given by the Eisenstein series in [4]. For example, if $m \in \mathbb{R}^{l},{ }^{t} m m \in \mathbb{N}$, and $s \in \mathbb{C}$ such that $\operatorname{Re}(s)>4$, then the Eisenstein series (with $k=0$ and $s \rightarrow s-\frac{1}{2}$ in [4])

$$
E_{m, s}(\tau, z):=\sum_{A \in \Gamma_{\infty}^{J}(K) \backslash \Gamma^{J}(K)}\left(\left.y^{s}\right|_{0, m} A\right)(\tau, z) \in J_{0, m, \lambda+, \lambda^{-}}
$$


with $\lambda^{+}=2^{t} m m(s-1)(s-2), \lambda^{-}=2^{t} \bar{m} \bar{m}(s-1)(s-2)$, and where $\Gamma_{\infty}^{J}(K)=$ $\left\{\left[\left(\begin{array}{cc}1 & \eta \\ 0 & 1\end{array}\right),(0, n)\right] \mid \eta, n \in \mathcal{O}_{K}\right\}$. Furthermore, for weights $k=1 / 2$ and $k=1$, the theta functions in [20] and [18] yield concrete examples of Jacobi forms on a subgroup of $\Gamma^{J}(K)$. It would be interesting to classify all theta functions which satisfy Definition 2.

Remark. At the end of the proof of Theorem 3.2 we explicitly state the moderate growth of Jacobi theta functions and of Jacobi forms that appear as lifts of modular forms of half-integral weight. Unfortunately, we cannot give such a growth condition for the Eisenstein series in (11), since we have not been able to completely determine its Fourier expansion (see also Section 3.2 of [4]).

\section{Lifting of modular forms to Jacobi forms}

In this section we provide new examples of Jacobi forms over $K=\mathbb{Q}(i)$ by lifting modular forms of half-integral weight over $K=\mathbb{Q}(i)$. Our construction is of the same flavor as the method of Kohnen [14] (see also Section 4 of Pitale [17]). It is likely that our results can be extended to other complex quadratic fields, but for simplicity we restrict to the case that $K=\mathbb{Q}(i), \mathcal{O}_{K}=\mathbb{Z}[i]$ is the ring of integers of $K$, and $\searrow_{K}=2 \mathbb{Z}[i]$ is the different of $K$.

3.1. Jacobi theta functions. Let $\mu \in \mathcal{R}$, where $\mathcal{R}$ is a complete system of representatives for the set of cosets $\frac{1}{2} \mathbb{Z}[i] / \mathbb{Z}[i]$. The theta function

$$
\theta_{\mu}(\tau, z):=\sum_{\omega \in \mathbb{Z}[i]} \exp \left\{\pi i \mathcal{T}_{\mathcal{R}}[(\omega+\mu) \tau(\omega+\mu)+2(\omega+\mu) z]\right\}
$$

is a crucial building block in our construction of Jacobi forms in Section 3.3. First we determine the transformation law of $\theta_{\mu}$ under translation and inversion.

Theorem 3.1. For every $\eta \in \mathbb{Z}[i]$ we have

$$
\theta_{\mu}(\tau+\eta, z)=\exp \left\{\pi i \mathcal{T}_{\mathcal{R}}\left[\eta \mu^{2}\right]\right\} \theta_{\mu}(\tau, z) .
$$

Moreover, we have

$$
\begin{aligned}
\theta_{\mu} & \left(-\tau^{-1}, \tau^{-1} z\right) \\
& =\frac{1}{2} \mathcal{N}(\tau)^{1 / 2} \exp \left\{\pi i \mathcal{T}_{\mathcal{R}}\left[\tilde{z} \tau^{-1} z\right]\right\} \sum_{\nu \in \mathcal{R}} \exp \left\{2 \pi i \mathcal{T}_{\mathcal{R}}[v \mu]\right\} \theta_{v}(\tau, z) .
\end{aligned}
$$

Proof. Equation (12) follows immediately from the definition of $\theta_{\mu}$. We apply Eichler's "embedding trick" to prove (13). Consider the following Jacobi theta function 
of higher degree:

$$
\Theta_{M}(Z, W):=\sum_{L \in \mathbb{Z}^{2}} \exp \left\{2 \pi i\left({ }^{t}(L+M) Z(L+M)+2^{t}(L+M) W\right)\right\},
$$

with $M \in \Re$, where $\Re$ is a complete system of representatives for the set of cosets $\frac{1}{2} \mathbb{Z}^{2} / \mathbb{Z}^{2}$, and where $Z$ and $W$ are variables in $\mathbb{H}_{2}$ (Siegel upper half plane of degree 2) and $\mathbb{C}^{2}$, respectively. Recall that

$$
\begin{aligned}
& \Theta_{M}\left(-Z^{-1}, Z^{-1} W\right) \\
& \quad=\frac{1}{2} \operatorname{det}(-i Z)^{\frac{1}{2}} \exp \left\{2 \pi i^{t} W Z^{-1} W\right\} \sum_{N \in \Re} \exp \left\{-4 \pi i{ }^{t} N M\right\} \Theta_{N}(Z, W),
\end{aligned}
$$

where $\operatorname{det}(-i Z)^{\frac{1}{2}}$ is positive if $Z=i Y$ for positive definite $Y \in \mathbb{R}^{(2,2)}$ (see [6] for a simple proof of (15) that does not require Poisson summation).

Now we will regard $\theta_{\mu}(\tau, z)$ as a special case of $\Theta_{M}(Z, W)$. Set $\Omega:=\left(\begin{array}{cc}1 & i \\ 1 & -i\end{array}\right)$. If $\tau=x+y \mathbf{k} \in \mathbb{H}_{\mathcal{Q}}, z \in \mathcal{Q}$, and $\mu \in \mathcal{R}$, then $Z:=\frac{1}{2}{ }^{t} \Omega\left(\begin{array}{cc}x & i y \\ i y & \bar{x}\end{array}\right) \Omega \in \mathbb{H}_{2}$, $W:=\frac{1}{2}{ }^{t} \Omega\left(\begin{array}{l}\|z\|_{\mathbb{C}} \\ \|z\|_{\overline{\mathbb{C}}}\end{array}\right) \in \mathbb{C}^{2}$, and $M:=\Omega^{-1}\left(\begin{array}{c}\mu \\ \bar{\mu}\end{array}\right)=\left(\begin{array}{l}\operatorname{Re} \mu \\ \operatorname{Im} \mu\end{array}\right) \in \Re$. Furthermore, if $\omega$ runs through $\mathbb{Z}[i]$, then $L:=\Omega^{-1}\left(\begin{array}{c}\omega \\ \bar{\omega}\end{array}\right)=\left(\begin{array}{c}\operatorname{Re} \omega \\ \operatorname{Im} \omega\end{array}\right)$ runs through $\mathbb{Z}^{2}$ and we find that

$$
\theta_{\mu}(\tau, z)=\Theta_{M}(Z, W) .
$$

As in [19] (see also [4]),

$$
(\tau, z) \mapsto\left(-\tau^{-1}, \tau^{-1} z\right) \in \mathbb{H}_{\mathcal{Q}} \times \mathcal{Q}
$$

corresponds to

$$
(Z, W) \mapsto\left(U\left(-Z^{-1}\right) U, U Z^{-1} W\right) \in \mathbb{H}_{2} \times \mathbb{C}^{2},
$$

where $U:=\left(\begin{array}{cc}1 & 0 \\ 0 & -1\end{array}\right)$. In particular,

$$
\theta_{\mu}\left(-\tau^{-1}, \tau^{-1} z\right)=\Theta_{M}\left(U\left(-Z^{-1}\right) U, U Z^{-1} W\right)=\Theta_{U M}\left(-Z^{-1}, Z^{-1} W\right) .
$$

Finally, we have

$$
\begin{aligned}
\mathcal{N}(\tau)^{1 / 2} & =\operatorname{det}(-i Z)^{1 / 2}, \\
\mathcal{T}_{\mathcal{R}}\left[\tilde{z} \tau^{-1} z\right] & =2{ }^{t} W Z^{-1} W, \\
\exp \left\{2 \pi i \mathcal{T}_{\mathcal{R}}[v \mu]\right\} & =\exp \left\{-2 \pi i \mathcal{T}_{\mathcal{R}}[v \mu]\right\}=\exp \left\{-4 \pi i^{t} N(U M)\right\},
\end{aligned}
$$

with $N:=\left(\begin{array}{l}\operatorname{Re} v \\ \operatorname{Im} v\end{array}\right) \in \Re$, so (13) follows from (15) and (17). 
3.2. Modular forms of half-integral weight. Elstrodt, Grunewald, and Mennicke [8] give a good overview of automorphic functions over complex quadratic fields $K$. We extend their notion of an automorphic form and we consider modular forms of half-integral weight over $K=\mathbb{Q}(i)$, and in particular, we introduce an analog of Kohnen's [14] plus space. Kojima [15] also discusses modular forms of half-integral weight over complex quadratic fields, but our point of view is quite different.

Set $\Gamma:=\mathrm{SL}_{2}(\mathbb{Z}[i])$ and $\Gamma_{0}(N):=\left\{\left(\begin{array}{l}* * \\ \gamma *\end{array}\right) \in \Gamma \mid \gamma \in N \mathbb{Z}[i]\right\}$. The theta function

$$
\theta(\tau):=\sum_{\omega \in \mathbb{Z}[i]} \exp \left\{\pi i \mathcal{T}_{\mathcal{R}}[\omega \tau \omega]\right\}
$$

is a modular form of weight $1 / 2$ on $\Gamma_{0}$ (4). More precisely, if $M=\left(\begin{array}{ll}* & * \\ \gamma & 8\end{array}\right) \in \Gamma_{0}$ (4), then

$$
\theta(M \circ \tau)=j(M, \tau) \theta(\tau)
$$

where $j(M, \tau):=\chi(M) \mathcal{N}(\gamma \tau+\delta)^{1 / 2}$ is the theta-multiplier. Here $\chi(M)$ is an eighth root of unity, which can be determined explicitly in some important special cases (see Section 4 of [19]): If $\mathcal{N}(\delta)=p$ is an odd rational prime, then

$$
\chi\left(\left(\begin{array}{ll}
* & * \\
\gamma & \delta
\end{array}\right)\right)=\left(\frac{\operatorname{Re}(\bar{\delta} \gamma)}{p}\right)
$$

is the Legendre symbol.

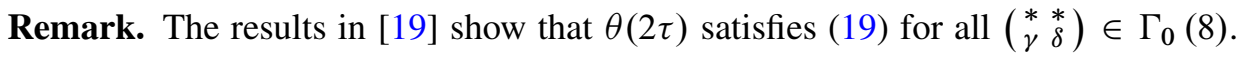
If, in addition, $\mathcal{N}(\delta)=p$ is a rational prime, then

$$
\chi\left(\left(\begin{array}{cc}
* * \\
\gamma & \delta
\end{array}\right)\right)=\left(\frac{2 \operatorname{Re}(\bar{\delta} \gamma)}{p}\right)
$$

which then easily implies (20).

We would like to address a small error in our paper [4], resulting from a mistake in [13]. We apply Proposition 4.6 of [13] to simplify (20), but unfortunately that proposition in [13] is not quite correct as stated. Also note a typographical error: The obvious factor of 2 in the theta multiplier of $\theta_{0}(2 \tau, z)$ (using the notation of Section 3.1) was left off in (14) of [4] (see (20) and (21) above).

Definition 3. A real-analytic function $h: \mathbb{H}_{\mathcal{Q}} \rightarrow \mathbb{C}$ is a modular form on $\Gamma_{0}(4)$ of weight $k-\frac{1}{2}(k \in \mathbb{N})$ and eigenvalue $\lambda \in \mathbb{C}$ if

(i) $h(M \circ \tau)=j(M, \tau)^{2 k-1} h(\tau)$ for all $M \in \Gamma_{0}(4)$,

(ii) $\Delta_{k-\frac{1}{2}} h=\lambda h$,

(iii) $h$ is of polynomial growth at the cusps of $\Gamma_{0}(4)$. 
We denote by $M_{k-\frac{1}{2}, \lambda}(4)$ the vector space of such modular forms of half-integral weight.

If $h \in M_{k-\frac{1}{2}, \lambda}(4)$, then $h(\tau+\eta)=h(\tau)$ for all $\eta \in \mathbb{Z}[i]$ and hence $h$ has a Fourier expansion of the form

$$
h(\tau)=\sum_{\omega \in \mathbb{Z}[i]} a_{y}(\omega) \exp \left\{\pi i \mathcal{T}_{\mathcal{R}}[\omega x]\right\} .
$$

Let $M_{k-\frac{1}{2}, \lambda}^{+}(4)$ denote the subspace of $M_{k-\frac{1}{2}, \lambda}(4)$, whose elements have Fourier expansions of the form

$$
h(\tau)=\sum_{\substack{\omega \in \mathbb{Z}[i] \\ \omega \equiv 0, \pm 1,2 i(\bmod 4)}} a_{y}(\omega) \exp \left\{\pi i \mathcal{T}_{\mathcal{R}}[\omega x]\right\} .
$$

Then $M_{k-\frac{1}{2}, \lambda}^{+}(4)$ is an analog of Kohnen's [14] plus space, and in contrast to [14] and [17] the plus space condition here does not depend on $k$.

Remark. The coefficients $a_{y}(\omega)$ in (22) can be described explicitly. Choose $s \in \mathbb{C}$ such that $\lambda=s^{2}-\left(k-\frac{3}{2}\right)^{2}$. Using (ii) and (iii) of Definition 3 and the properties of the usual Bessel function $K_{s}$, one can show as in [8] (pages 105-106) that the right hand side of (22) can be written as

$$
c(y)+y^{\frac{3}{2}-k} \sum_{\omega \in \mathbb{Z}[i] \backslash\{0\}} a(\omega) K_{s}(2 \pi|\omega| y) \exp \left\{\pi i \mathcal{T}_{\mathcal{R}}[\omega x]\right\},
$$

where the coefficients $a(\omega) \in \mathbb{C}$ are independent of $y$ and

$$
c(y):=a_{0} y^{\frac{3}{2}-k+s}+b_{0} y^{\frac{3}{2}-k-s}+c_{0} \delta(s) y^{\frac{3}{2}-k} \log (y),
$$

with $a_{0}, b_{0}, c_{0} \in \mathbb{C}$, and $\delta(s):= \begin{cases}0 & \text { if } s \neq 0, \\ 1 & \text { if } s=0 .\end{cases}$

3.3. The lift. We are now in a position to state the main result of this Section, which gives a lift from $M_{k-\frac{1}{2}, \lambda}^{+}(4)$ to $J_{k, \frac{1}{\sqrt{2}}, \Lambda, \Lambda}$ with $\Lambda:=\lambda+k^{2}-k+1$.

Theorem 3.2. Let $h=\sum a_{y}(\omega) \exp \left\{\pi i \mathcal{T}_{\mathcal{R}}[\omega x]\right\} \in M_{k-\frac{1}{2}, \lambda}^{+}(4)$ and write

$$
\begin{aligned}
h(\tau) & =\sum_{\nu \in\{0, \pm 1,2 i\}} h^{(v)}(4 \tau) \\
& =\sum_{\mu \in \mathcal{R}} h_{\mu}(4 \tau)
\end{aligned}
$$


with

$$
h^{(v)}(\tau):=\sum_{\omega \in \mathbb{Z}[i]} a_{\frac{y}{4}}(4 \omega+v) \exp \left\{\frac{\pi i}{4} \mathcal{T}_{\mathcal{R}}[(4 \omega+v) x]\right\}
$$

and

$$
h_{0}:=h^{(0)}, h_{\frac{1}{2}}:=h^{(-1)}, h_{\frac{i}{2}}:=h^{(1)} \text {, and } h_{\frac{1+i}{2}}:=h^{(2 i)} .
$$

Then for $\mu \in \mathcal{R}$ we have

$$
h_{\mu}(\tau+\eta)=\exp \left\{-\pi i \mathcal{T}_{\mathcal{R}}\left[\eta \mu^{2}\right]\right\} h_{\mu}(\tau)
$$

for every $\eta \in \mathbb{Z}[i]$ and also

$$
h_{\mu}\left(-\tau^{-1}\right)=\frac{1}{2} \mathcal{N}(\tau)^{k-1 / 2} \sum_{\nu \in \mathcal{R}} \exp \left\{2 \pi i \mathcal{T}_{\mathcal{R}}[\nu \mu]\right\} h_{\nu}(\tau) .
$$

We have

$$
\phi(\tau, z):=\sum_{\mu \in \mathcal{R}} h_{\mu}(\tau) \theta_{\mu}(\tau, z) \in J_{k, \frac{1}{\sqrt{2}}, \Lambda, \Lambda}
$$

with $\Lambda:=\lambda+k^{2}-k+1$.

Proof. Equation (24) follows directly from the definition of the $h_{\mu}$.

We turn to the proof of (25). It is not hard to see that we can write

$$
h^{(\nu)}(\tau)=\frac{1}{16} \sum_{n(\bmod 4)} \exp \left\{-\frac{\pi i}{4} \mathcal{T}_{\mathcal{R}}[n \nu]\right\} h\left(\frac{\tau+n}{4}\right) .
$$

To determine the behavior of this function under inversion, we split the sum over $n$ into two groups:

$$
\begin{aligned}
& S_{1}:=\left\{n_{1}+i n_{2} ; n_{1} \equiv n_{2}(\bmod 2), n_{1}, n_{2}(\bmod 4)\right\} \\
& S_{2}:=\left\{n_{1}+i n_{2} ; n_{1} \equiv 1+n_{2}(\bmod 2), n_{1}, n_{2}(\bmod 4)\right\} .
\end{aligned}
$$

Clearly

$$
h^{(v)}\left(-(4 \tau)^{-1}\right)=g_{\nu, 1}(\tau)+g_{\nu, 2}(\tau),
$$

where

$$
\begin{aligned}
g_{\nu, 1}(\tau) & :=\frac{1}{16} \sum_{n \in S_{1}} \exp \left\{-\frac{\pi i}{4} \mathcal{T}_{\mathcal{R}}[n \nu]\right\} h\left(\frac{1}{4}\left((-4 \tau)^{-1}+n\right)\right), \\
g_{\nu, 2}(\tau) & :=\frac{1}{16} \sum_{n \in S_{2}} \exp \left\{-\frac{\pi i}{4} \mathcal{T}_{\mathcal{R}}[n \nu]\right\} h\left(\frac{1}{4}\left((-4 \tau)^{-1}+n\right)\right) .
\end{aligned}
$$


We first consider $g_{\nu, 1}(\tau)$. Write

$$
\begin{aligned}
& g_{\nu, 1}\left(-\frac{\tau^{-1}}{4}\right)=\quad \frac{1}{16} \sum_{n \in S_{1}} \exp \left\{-\frac{\pi i}{4} \mathcal{T}_{\mathcal{R}}[n \nu]\right\} h\left(\frac{1}{4}(\tau+n)\right) \\
& =\frac{1}{16} \sum_{\substack{\omega \in \mathbb{Z}[i] \\
\omega \equiv 0, \pm 1,2 i(\bmod 4)}} a_{\frac{y}{4}}(\omega) \exp \left\{\frac{\pi i}{4} \mathcal{T}_{\mathcal{R}}[x \omega]\right\} \sum_{n \in S_{1}} \exp \left\{\frac{\pi i}{4} \mathcal{T}_{\mathcal{R}}[n(\omega-v)]\right\} .
\end{aligned}
$$

The sum over $n$ on the right hand side of (28) equals

$$
\begin{aligned}
& \sum_{n(\bmod 2)} \exp \left\{\frac{\pi i}{4} \mathcal{T}_{\mathcal{R}}[2 n(\omega-v)]\right\}+\sum_{n(\bmod 2)} \exp \left\{\frac{\pi i}{4} \mathcal{T}_{\mathcal{R}}[(2 n+1-i)(\omega-v)]\right\} \\
= & \left(1+\exp \left\{\frac{\pi i}{4} \mathcal{T}_{\mathcal{R}}[(1-i)(\omega-v)]\right\}\right) \sum_{n(\bmod 2)} \exp \left\{\frac{\pi i}{4} \mathcal{T}_{\mathcal{R}}[2 n(\omega-v)]\right\},
\end{aligned}
$$

where the sum over $n$ on the right hand side of (29) vanishes unless $\omega \equiv v(\bmod 2)$. If $\omega \equiv v(\bmod 2)$, then the plus space condition forces the first factor on the right hand side of (29) to be 0 unless $\omega \equiv v(\bmod 4)$. Hence

$$
g_{\nu, 1}\left(-\frac{\tau^{-1}}{4}\right)=\frac{1}{2} h^{(v)}(\tau)
$$

and thus

$$
\frac{1}{2} h^{(v)}\left(-(4 \tau)^{-1}\right)=g_{v, 2}(\tau)
$$

We next consider $g_{v, 2}(\tau)$. Clearly

$$
S_{2}=\{1,3, i, 3 i, 1+2 i, i+2,2+3 i, 3+2 i\} .
$$

Observing that $h$ is invariant under translations, one can check that

$$
g_{\nu, 2}(\tau)=\frac{1}{16} \sum_{n \in S_{2}} \exp \left\{-\frac{\pi i}{4} \mathcal{T}_{\mathcal{R}}[n v]\right\} h\left(M_{n} \circ\left(\tau-\frac{\bar{n}}{4}\right)\right),
$$

where the matrices $M_{n}$ are defined as follows:

$$
\begin{gathered}
M_{1}=\left(\begin{array}{ll}
1 & 0 \\
4 & 1
\end{array}\right), \quad M_{3}=\left(\begin{array}{ll}
3 & -1 \\
4 & -1
\end{array}\right), \quad M_{i}=\left(\begin{array}{cc}
i & 0 \\
4 & -i
\end{array}\right), \\
M_{3 i}=\left(\begin{array}{cc}
3 i-1 \\
4 & i
\end{array}\right), \quad M_{1+2 i}=\left(\begin{array}{cc}
1+2 i & 1 \\
4 & 1-2 i
\end{array}\right), \quad M_{i+2}=\left(\begin{array}{cc}
2+i & 1 \\
4 & 2-i
\end{array}\right), \\
M_{2+3 i}=\left(\begin{array}{cc}
2+3 i & 3 \\
4 & 2-3 i
\end{array}\right), \quad M_{3+2 i}=\left(\begin{array}{cc}
3+2 i & 3 \\
4 & 3-2 i
\end{array}\right) .
\end{gathered}
$$


If $M_{n}=\left(\begin{array}{ll}a & b \\ c & d\end{array}\right)$ is from the list above, then the transformation law of $h$ implies that

$$
h\left(M_{n} \circ \tau\right)=\epsilon_{n} \mathcal{N}(c \tau+d)^{k-\frac{1}{2}} h(\tau)
$$

with

$$
\epsilon_{n}:= \begin{cases}1 & \text { if } n \in\{1,3, i, 3 i, 1+2 i, 3+2 i\}, \\ -1 & \text { if } n \in\{i+2,2+3 i\} .\end{cases}
$$

Writing $v=v_{1}+i v_{2}$ and $n=n_{1}+i n_{2}$, we find that

$$
\begin{aligned}
g_{v, 2}(\tau)= & \frac{\mathcal{N}^{k-\frac{1}{2}}(4 \tau)}{16}\left[\exp \left\{-\frac{2 \pi i}{4} v_{1}\right\} h\left(\tau-\frac{1}{4}\right)+\exp \left\{\frac{2 \pi i}{4} v_{1}\right\} h\left(\tau+\frac{1}{4}\right)\right. \\
& +\exp \left\{\frac{2 \pi i}{4} v_{2}\right\} h\left(\tau+\frac{i}{4}\right)+\exp \left\{-\frac{2 \pi i}{4} \nu_{2}\right\} h\left(\tau-\frac{i}{4}\right) \\
& +\exp \left\{\frac{2 \pi i}{4}\left(-v_{1}+2 v_{2}\right)\right\} h\left(\tau+\frac{2 i-1}{4}\right) \\
& -\exp \left\{\frac{2 \pi i}{4}\left(-2 v_{1}+v_{2}\right)\right\} h\left(\tau+\frac{i-2}{4}\right) \\
& -\exp \left\{\frac{2 \pi i}{4}\left(-2 v_{1}-v_{2}\right)\right\} h\left(\tau+\frac{3 i-2}{4}\right) \\
& \left.+\exp \left\{\frac{2 \pi i}{4}\left(v_{1}+2 v_{2}\right)\right\} h\left(\tau+\frac{2 i+1}{4}\right)\right] \\
= & \mathcal{N}^{k-\frac{1}{2}}(4 \tau) \\
& 16 \quad \sum \exp \left\{\frac{2 \pi i}{4}\left(-v_{1}-n_{1}\right)\right\}+\exp \left\{\frac{2 \pi i}{4}\left(v_{1}+n_{1}\right)\right\} \\
& +\exp \left\{\frac{2 \pi i}{4}\left(v_{2}-n_{2}\right)\right\}+\exp \left\{\frac{2 \pi i}{4}\left(-v_{2}+n_{2}\right)\right\} \\
& +\exp \left\{\frac{2 \pi i}{4}\left(-v_{1}+2 v_{2}-n_{1}-2 n_{2}\right)\right\} \\
& -\exp \left\{\frac{2 \pi i}{4}\left(-2 v_{1}+v_{2}-2 n_{1}-n_{2}\right)\right\} \\
& -\exp \left\{\frac{2 \pi i}{4}\left(-2 v_{1}-v_{2}-2 n_{1}+n_{2}\right)\right\} \\
& \left.+\exp \left\{\frac{2 \pi i}{4}\left(v_{1}+2 v_{2}+n_{1}-2 n_{2}\right)\right\}\right] h^{(n)}(4 \tau),
\end{aligned}
$$

where the last equation follows after inserting the Fourier expansion of $h(\tau)$. 
Specifically,

$$
\begin{gathered}
g_{0,2}(\tau)=\frac{\mathcal{N}^{k-\frac{1}{2}}(4 \tau)}{4}\left(h^{(0)}(4 \tau)+h^{(1)}(4 \tau)+h^{(-1)}(4 \tau)+h^{(2 i)}(4 \tau)\right), \\
g_{1,2}(\tau)=\frac{\mathcal{N}^{k-\frac{1}{2}}(4 \tau)}{4}\left(h^{(0)}(4 \tau)-h^{(1)}(4 \tau)+h^{(-1)}(4 \tau)-h^{(2 i)}(4 \tau)\right), \\
g_{-1,2}(\tau)=\frac{\mathcal{N}^{k-\frac{1}{2}}(4 \tau)}{4}\left(h^{(0)}(4 \tau)+h^{(1)}(4 \tau)-h^{(-1)}(4 \tau)-h^{(2 i)}(4 \tau)\right), \\
g_{2 i, 2}(\tau)=\frac{\mathcal{N}^{k-\frac{1}{2}}(4 \tau)}{4}\left(h^{(0)}(4 \tau)-h^{(1)}(4 \tau)-h^{(-1)}(4 \tau)+h^{(2 i)}(4 \tau)\right),
\end{gathered}
$$

and using (30) together with the substitution $\tau \rightarrow \frac{\tau}{4}$ gives (25).

Now we will show that $\phi$ defined by (26) satisfies

$$
\left(\left.\phi\right|_{k, \frac{1}{\sqrt{2}}} A\right)(\tau, z)=\phi(\tau, z)
$$

for all $A \in \Gamma^{J}(K)$. For $A=\left[\left(\begin{array}{ll}1 & 0 \\ 0 & 1\end{array}\right),(\lambda, \mu)\right]$ this follows directly from the definition of $\theta_{\mu}$. Equations (12), (13), (24), and (25) imply that (31) holds for $A=$ $\left[\left(\begin{array}{ll}1 & \eta \\ 0 & 1\end{array}\right),(0,0)\right]$ and $A=\left[\left(\begin{array}{cc}0 & -1 \\ 1 & 0\end{array}\right),(0,0)\right]$. Recall that the matrices $\left(\begin{array}{ll}1 & \eta \\ 0 & 1\end{array}\right)$ for all $\eta \in \mathbb{Z}[i]$ and $\left(\begin{array}{cc}0 & -1 \\ 1 & 0\end{array}\right)$ generate $\mathrm{SL}_{2}(\mathbb{Z}[i])$. Hence (31) holds for all $A \in \Gamma^{J}(K)$.

Note that $\Delta_{k-\frac{1}{2}} h=\lambda h$ implies that $\Delta_{k-\frac{1}{2}} h_{\mu}=\lambda h_{\mu}$. A lengthy but straightforward computation (using Mathematica) reveals that

$$
\begin{aligned}
\bigodot_{k, \frac{1}{\sqrt{2}}}^{ \pm}\left(h_{\mu} \theta_{\mu}\right)=y^{-k} \bigodot_{0, \frac{1}{\sqrt{2}}}^{ \pm}\left(y^{k} h_{\mu} \theta_{\mu}\right) & =\left(\frac{3}{4} h_{\mu}+y^{-k+\frac{1}{2}} \Delta_{0}\left(y^{k-\frac{1}{2}} h_{\mu}\right)\right) \theta_{\mu} \\
& \stackrel{(8)}{=}\left(\frac{3}{4} h_{\mu}+\Delta_{k-\frac{1}{2}}\left(h_{\mu}\right)+\left(k-\frac{1}{2}\right)^{2} h_{\mu}\right) \theta_{\mu} \\
& =\left(\lambda+k^{2}-k+1\right) h_{\mu} \theta_{\mu} .
\end{aligned}
$$

Finally, $\phi$ is also of moderate growth. More precisely,

$$
\exp \left\{-2 \pi \frac{|v|^{2}}{y}\right\}\left|\exp \left\{\pi i \mathcal{T}_{\mathcal{R}}[(\omega+\mu) \tau(\omega+\mu)+2(\omega+\mu) z]\right\}\right|=\exp \{-2 \pi y a\}
$$

with

$$
a:=\left(|\omega+\mu|-\frac{|v|}{y}\right)^{2}+\frac{2}{y}(|(\omega+\mu) v|+\operatorname{Re}((\omega+\mu) v)) \geq 0 .
$$

So $\theta_{\mu}(\tau, z)=O\left(\exp \left\{2 \pi|v|^{2} / y\right\}\right)$ as $y \rightarrow \infty$ and $\phi(\tau, z)=O\left(y^{N} \exp \left\{2 \pi|v|^{2} / y\right\}\right)$ for some $N>0$ as $y \rightarrow \infty$. We conclude that $\phi \in J_{k, \frac{1}{\sqrt{2}}, \Lambda, \Lambda}$ with $\Lambda=\lambda+k^{2}-$ $k+1$. 
Remarks. (1) A half-integral weight cusp form over $K=\mathbb{Q}(i)$ has a Fourier expansion as in (23) with constant coefficient $c(y)=0$. Therefore if $h \in M_{k-\frac{1}{2}, \lambda}^{+}(4)$ is a cusp form, then $h$ is of exponential decay in $y$ and one finds that $\phi$ in (26) satisfies the Jacobi cusp form condition (10). Hence the lift in (26) preserves cuspidality.

(2) The Jacobi theta functions of higher degree $\Theta_{M}(Z, W)$ in (14) are linearly independent as functions of $W$ for fixed $Z$ (see Lemma 3.1 of Ziegler [25]). Equation (16) implies that the functions $z \rightarrow \theta_{\mu}(\tau, z)\left(\mu=0, \frac{1}{2}, \frac{i}{2}, \frac{1+i}{2}\right)$ are linearly independent for every $\tau \in \mathbb{H}_{\mathcal{Q}}$, and thus the lift in (26) is injective.

(3) It would be interesting to explore a Hecke theory for Jacobi forms over complex quadratic fields, which would allow one to investigate if the lift in (26) sends Hecke eigenforms to Hecke eigenforms.

(4) The real-analytic Jacobi forms in [17] can essentially all be obtained from half-integral weight Maass forms (see the Remark after Theorem 4.6 in [17]). We thank Ralf Schmidt for pointing out that an analogous statement should also hold in our setting here.

We end this section with an explicit example of Theorem 3.2. We have $\theta \in$ $M_{\frac{1}{2}, 0}^{+}(4)$. We find that $\theta(\tau)=\theta(i \tau i)=\sum_{\mu \in \mathcal{R}} h_{\mu}(4 \tau)$ with $h_{\mu}(\tau):=\theta_{\mu}(i \tau i, 0)$. Note that $i \tau i=-x+y \mathbf{k} \in \mathbb{H}_{\mathcal{Q}}, h_{\mu}(\tau)=h_{\mu}(i \tau i)$ for $\mu=0$ and $\mu=\frac{1+i}{2}$, and $h_{\frac{1}{2}}(\tau)=h_{\frac{i}{2}}(i \tau i)$. Then $h_{\mu}$ satisfies (24) and (25) with $k=1$ and $\Delta_{\frac{1}{2}} h_{\mu}=0$. Hence

$$
\sum_{\mu \in \mathcal{R}} \theta_{\mu}(i \tau i, 0) \theta_{\mu}(\tau, z) \in J_{1, \frac{1}{\sqrt{2}}, \frac{3}{4}, \frac{3}{4}} .
$$

\section{Algebras of invariant differential operators}

We now turn to the Lie-theoretic part of the paper, the purpose of which is to prove Theorem 2.3. Fix throughout this section a real Lie group $G$, a closed subgroup $K$, and a representation $\pi$ of $K$ on a complex vector space $V$. The $G$-vector bundle $G \times \times_{K} V$ over $G / K$ is the set of equivalence classes $[g, v]:=\left\{\left(g k^{-1}, \pi(k) v\right) \in\right.$ $G \times V: k \in K\}$, with $G$-action $g^{\prime}[g, v]:=\left[g^{\prime} g, v\right]$ and projection $[g, v] \mapsto g K$. We will write $C_{\text {sec }}^{\infty}\left(G \times_{K} V\right)$ for the space of smooth sections of this bundle, and $\lambda$ for the canonical action of $G$ on it:

$$
(\lambda(g)(s))(x K):=g s\left(g^{-1} x K\right) .
$$

Definition 4. Let $\mathbb{D}\left(G \times{ }_{K} V\right)$ be the algebra of $G$-invariant differential operators on $G \times_{K} V$.

In this section we give a Lie algebraic description of $\mathbb{D}\left(G \times_{K} V\right)$. This generalizes a result of Helgason [11] giving such a description in the case that $K$ is connected and 
the inclusion $\mathfrak{k} \subseteq \mathrm{g}$ is $K$-split. The generalization is not major, but to our knowledge it has not appeared previously. In fact, Helgason's result suffices for the purposes of the present paper, but the general result is of independent interest.

Given any real Lie group $H$, let $\mathfrak{h}_{0}$ be its real Lie algebra, and let $\mathfrak{h}$ be the complexification $\mathfrak{h}_{0} \otimes_{\mathbb{R}} \mathbb{C}$ of $\mathfrak{h}_{0}$. Given any representation $\sigma$ of $H$ on a complex vector space $W$, write $W^{\sigma(H)}$ and $W^{\sigma(\mathfrak{h})}$ for the $H$ - and $\mathfrak{h}$-invariant vectors in $W$, respectively. When $\sigma$ is clear from the context, we write simply $W^{H}$ and $W^{\mathfrak{h}}$. Write $S^{n}(W)$ for the $n^{\text {th }}$ symmetric power of $W$, also an $H$-module, and $\delta(W)$ for the symmetric algebra $\bigoplus_{n=0}^{\infty} \delta^{n}(W)$. Let degree: $\delta(W) \rightarrow S(W)$ be the endomorphism which acts on $S^{n}(W)$ by $n$.

Let $\mathfrak{U}(\mathfrak{h})$ be the universal enveloping algebra of $\mathfrak{h}$, with degree filtration $\mathfrak{U}^{r}(\mathfrak{h})$. Let $3(\mathfrak{h})$ denote its center, which is of course $\mathfrak{U}(\mathfrak{h})^{\mathfrak{h}}$. The transpose antiautomorphism $\Theta \mapsto \Theta^{T}$ of $\mathcal{U}(\mathfrak{h})$ is $\left(X_{1} \cdots X_{n}\right)^{T}:=(-1)^{n} X_{n} \cdots X_{1}$. It preserves 3(h) .

Let Sym: $S(\mathfrak{h}) \rightarrow \mathcal{U}(\mathfrak{h})$ be the symmetrizer map, a filtration-preserving $H$ equivalence carrying $X_{1} \cdots X_{n}$ to $\frac{1}{n !} \sum_{v \in S_{n}} X_{v(1)} \cdots X_{v(n)}$. Note that $\operatorname{Sym}(\Omega)^{T}=$ $(-1)^{\text {degree }(\Omega)} \operatorname{Sym}(\Omega)$. Also, Sym restricts to a linear bijection from $S(\mathfrak{h})^{\mathfrak{h}}$ to $3(\mathfrak{h})$.

We will always use $\lambda, \rho$, and ad for the left, right, and adjoint actions, respectively, in any context. Thus we have these three actions of $H$ on $C^{\infty}(H)$, and of $\mathfrak{h}$ and therefore also of $\mathfrak{U}(\mathfrak{h})$ on both $C^{\infty}(H)$ and $\mathfrak{U}(\mathfrak{h})$. In addition, we have the action ad of $H$ on $\mathfrak{U}(\mathfrak{h})$. All of these actions are compatible in the usual sense, and the adjoint action on $\mathcal{U}(\mathfrak{h})$ preserves its degree filtration. It is useful to note that for $f \in C^{\infty}(H)$ and $\Theta \in \mathcal{U}(\mathfrak{h})$,

$$
(\rho(\Theta) f)(e)=\left(\lambda\left(\Theta^{T}\right) f\right)(e) .
$$

Similarly, if $(\sigma, W)$ is any representation of $H$, we denote by $\lambda_{\sigma}, \rho_{\sigma}$, and $\operatorname{ad}_{\sigma}$ the corresponding left, right, and adjoint actions of $H, \mathfrak{h}$, and $\mathfrak{U}(\mathfrak{h})$ on $\operatorname{End}(W)$.

Returning to the representation $(\pi, V)$ of $K \subseteq G$ on $V$, let us define

$$
\begin{aligned}
\mathcal{E}_{\pi} & :=\mathcal{U}(\mathfrak{g}) \otimes \operatorname{End}(V), \\
\mathcal{I}_{\pi} & :=\mathcal{E}_{\pi}\{Y \otimes 1+1 \otimes \pi(Y): Y \in \mathfrak{F}\}, \\
\mathcal{A}_{\pi} & :=\left\{P \in \mathcal{E}_{\pi}: \mathcal{I}_{\pi} P \subseteq \mathcal{I}_{\pi}\right\}, \\
\mathcal{D}_{\pi}: & =\left(\mathcal{E}_{\pi} / \mathcal{I}_{\pi}\right)^{\left(\overline{\operatorname{ad} \otimes \mathrm{ad}_{\pi}}\right)(K)}=\left(\mathcal{A}_{\pi} / \mathcal{I}_{\pi}\right)^{\left(\overline{\operatorname{ad} \otimes \mathrm{ad}_{\pi}}\right)\left(K / K_{e}\right)} .
\end{aligned}
$$

Some explanation is necessary. First, $\varepsilon_{\pi}$ is an associative algebra possessing three natural $K$-actions, namely $\lambda \otimes \lambda_{\pi}, \rho \otimes \rho_{\pi}$, and ad $\otimes \operatorname{ad}_{\pi}$. Second, $\mathcal{I}_{\pi}$ is the left ideal $\left(\rho \otimes \rho_{\pi}\right)(\mathfrak{F})\left(\mathcal{E}_{\pi}\right)$ of $\mathcal{E}_{\pi}$. Third, $\mathcal{A}_{\pi}$ is a subalgebra of $\mathcal{E}_{\pi}$ within which $\mathcal{I}_{\pi}$ is a two-sided ideal, so $\mathcal{A}_{\pi} / \mathcal{I}_{\pi}$ is an associative algebra. Fourth, ad $\otimes \mathrm{ad}_{\pi}$ preserves both $\mathcal{A}_{\pi}$ and $\mathcal{I}_{\pi}$ and acts by algebra automorphisms. Therefore there is a quotient action $\overline{\operatorname{ad} \otimes \operatorname{ad}_{\pi}}$ of $K$ on $\mathcal{E}_{\pi} / \mathcal{I}_{\pi}$ which preserves $\mathcal{A}_{\pi} / \mathcal{I}_{\pi}$ and acts on it by automorphisms. Thus $\mathscr{D}_{\pi}$ is an associative algebra. All four spaces inherit a filtration from $\mathcal{U}(\mathfrak{g})$. 
Check that $\mathcal{A}_{\pi}$ is the pull-back of the $\left(\overline{\operatorname{ad} \otimes \operatorname{ad}_{\pi}}\right)(\mathfrak{k})$-invariants of $\mathcal{E}_{\pi} / \mathcal{I}_{\pi}$, i.e., $\mathcal{A}_{\pi} / \mathcal{I}_{\pi}$ is $\left(\mathcal{E}_{\pi} / \mathcal{I}_{\pi}\right)^{\mathfrak{k}}$. In particular, the identity component $K_{e}$ of $K$ acts trivially on $\mathcal{A}_{\pi} / \mathcal{I}_{\pi}$, so we may regard $\overline{\operatorname{ad} \otimes \mathrm{ad}_{\pi}}$ as an action of the discrete group $K / K_{e}$ on it. This gives the second form of $\mathscr{D}_{\pi}$. Note that if $K$ is connected, then $\mathscr{D}_{\pi}=$ $\left(\mathcal{E}_{\pi} / \mathcal{I}_{\pi}\right)^{\mathfrak{F}}=\mathcal{A}_{\pi} / \mathcal{I}_{\pi}$.

For future reference, observe that for $P \in \mathcal{E}_{\pi}$ and $\Theta \in \mathcal{U}(\mathfrak{F})$,

$$
P \cdot(\Theta \otimes 1) \equiv P \cdot\left(1 \otimes \pi\left(\Theta^{T}\right)\right) \text { modulo } \mathcal{I}_{\pi} .
$$

The main result of this section is that $\mathbb{D}\left(G \times_{K} V\right)$ and $\mathcal{D}_{\pi}$ are isomorphic algebras. In order to give the isomorphism explicitly we need a few remarks. Observe that the actions $\lambda \otimes 1$ of $G$ and $\rho \otimes \pi$ of $K$ on $C^{\infty}(G) \otimes V$ commute. Hence $\lambda \otimes 1$ is a $G$-action on $\left(C^{\infty}(G) \otimes V\right)^{(\rho \otimes \pi)(K)}$, the space of $K$-invariant $V$-valued smooth functions on $G$. In concrete terms, the invariance condition is $\pi(k) f(g k)=f(g)$ for all $g \in G$ and $k \in K$.

Henceforth we will use the following well-known $G$-identification:

$$
C_{\mathrm{sec}}^{\infty}\left(G \times{ }_{K} V\right) \equiv\left(C^{\infty}(G) \otimes V\right)^{(\rho \otimes \pi)(K)} ;
$$

a function $f$ on the right corresponds to the section $s_{f}(g K):=[g, f(g)]$.

Let us write $\beta$ for the defining action of the associative algebra $\operatorname{End}(V)$ on $V$, and $\rho \otimes \beta$ for the outer tensor product action of $\varepsilon_{\pi}$ on $C^{\infty}(G) \otimes V$ built from $\rho$ and $\beta$. Note that the restriction of $\rho \otimes \beta$ to $\mathcal{I}_{\pi}$ annihilates $C_{\mathrm{sec}}^{\infty}\left(G \times{ }_{K} V\right)$, so $\rho \otimes \beta$ factors through to a map

$$
\overline{\rho \otimes \beta}: \mathcal{E}_{\pi} / \mathcal{I}_{\pi} \rightarrow \operatorname{Hom}\left[C_{\mathrm{sec}}^{\infty}\left(G \times_{K} V\right), C^{\infty}(G) \otimes V\right] .
$$

Theorem 4.1. The restriction of $\overline{\rho \otimes \beta}$ to $\mathcal{D}_{\pi}$ is an algebra isomorphism from $\mathcal{D}_{\pi}$ to $\mathbb{D}\left(G \times_{K} V\right)$ which carries the $\mathcal{U}(\mathrm{g})$-filtration on $\mathfrak{D}_{\pi}$ to the order filtration.

Proof. Given $P \in \mathcal{E}_{\pi}$, write $\bar{P}$ for its image in $\mathcal{E}_{\pi} / \mathcal{I}_{\pi}$. First, we claim that if $\bar{P} \in D_{\pi}$, then $\overline{\rho \bigotimes \beta}(\bar{P})$ maps $C_{\text {sec }}^{\infty}\left(G \times_{K} V\right)$ into itself. This is a consequence of the following fact: for any $\Theta \in \mathcal{U}(\mathfrak{g}), T \in \operatorname{End}(V)$, and $k \in K$,

$$
(\rho \otimes \pi)(k) \circ(\rho \otimes \beta)(\Theta \otimes T)=(\rho \otimes \beta)\left(\left(\operatorname{ad} \otimes \operatorname{ad}_{\pi}\right)(k)(\Theta \otimes T)\right) \circ(\rho \otimes \pi)(k) .
$$

Clearly $\rho \otimes \beta$ bijects $\mathcal{E}_{\pi}$ to the space of left $G$-invariant differential operators on the $G$-bundle $G \times V$ over $G$. It follows that $\overline{\rho \otimes \beta}$ carries $\mathcal{D}_{\pi}$ to $\mathbb{D}\left(G \times_{K} V\right)$. Since $\rho \otimes \beta$ is an algebra homomorphism, $\left.\overline{\rho \otimes \beta}\right|_{D_{\pi}}$ is one also.

It remains to prove that $\overline{\rho \otimes \beta}$ bijects $\mathscr{D}_{\pi}$ to $\mathbb{D}\left(G \times_{K} V\right)$. In order to prove injectivity, let $\mathfrak{m}$ be any subspace of $\mathfrak{g}$ complementary to $\mathfrak{k}$. It follows from the Poincaré-Birkhoff-Witt theorem that the subspace $\operatorname{Sym}(S(\mathfrak{m})) \otimes \operatorname{End}(V)$ of $\mathcal{E}_{\pi}$ is complementary to $\mathcal{I}_{\pi}$. An argument using coordinates near $e$ shows that if $P$ is a 
non-zero element of $\operatorname{Sym}(S(\mathfrak{m})) \otimes \operatorname{End}(V)$, then $\overline{\rho \otimes \beta}(\bar{P})$ is non-zero. This proves that $\overline{\rho \otimes \beta}$ is injective on $\mathcal{E}_{\pi} / \mathcal{I}_{\pi}$.

To prove surjectivity, suppose that $D$ is any element of $\mathbb{D}\left(G \times_{K} V\right)$. Let us write eval $e_{e}$ for the map which carries any function on $G$ to its value at $e$, and $D_{e}$ for eval $_{e} \circ D$. Clearly $D$ is determined by $D_{e}$, so it will suffice to find $\bar{P} \in \mathscr{D}_{\pi}$ such that $\overline{\rho \otimes \beta}(\bar{P})_{e}=D_{e}$. Note that $D_{e}$ is $K$-invariant in the natural sense.

Again using coordinates at $e$, extend $D_{e}$ to a $V$-valued differential operator $\delta$ on $C^{\infty}(G) \otimes V$ supported at $e$. By the first sentence of the second paragraph of the proof, there is an element $P$ of $\mathcal{E}_{\pi}$ such that $(\rho \otimes \beta)(P)_{e}=\delta$, and in particular, $\overline{\rho 区 \beta}(\bar{P})_{e}=D_{e}$. Now use the third paragraph of the proof to verify that $\bar{P} \mapsto$ $\overline{\rho \otimes \beta}(\bar{P})_{e}$ is a $K$-injection from $\mathcal{E}_{\pi} / \mathcal{I}_{\pi}$ to the space of differential operators on $G \times \times_{K} V$ supported at $e K$. Together with the $K$-invariance of $D_{e}$, this yields $\bar{P} \in$ $\left(\mathcal{E}_{\pi} / \mathcal{I}_{\pi}\right)^{\overline{\mathrm{ad} \otimes \mathrm{ad}_{\pi}}(K)}$.

Finally, check that $\mathcal{A}_{\pi}$ is the pull-back to $\mathcal{E}_{\pi}$ of $\left(\mathcal{E}_{\pi} / \mathcal{I}_{\pi}\right)^{\overline{\mathrm{ad} \otimes \mathrm{ad}_{\pi}}(\mathfrak{F})}$. Therefore $D_{\pi}$ is in fact $\left(\mathcal{E}_{\pi} / \mathcal{I}_{\pi}\right)^{\overline{\mathrm{ad} \otimes \operatorname{ad} \pi}(K)}$. The filtration-preserving statement is clear.

It is interesting to observe that $\mathscr{D}_{\pi}$ is closely related to the step algebra defined by Mickelsson [16]. Indeed, for $G$ a complex reductive group, $H$ a connected complex reductive subgroup of $G, K$ the unipotent radical of a Borel subgroup of $H$, and $V$ trivial, $D_{\pi}$ is precisely the step algebra of the pair $(G, H)$.

4.1. Centers. Assume in this section that $K$ is connected. Regarding $\mathfrak{U}(\mathfrak{g})$ as the subalgebra $\mathfrak{U}(\mathfrak{g}) \otimes 1$ of $\mathcal{E}_{\pi}$, its center $3(\mathfrak{g})$ is clearly also the center of $\mathcal{E}_{\pi}$. In particular, $3(\mathrm{~g})$ is contained in $\mathcal{E}_{\pi}^{K}$, and so the canonical map $\Theta \mapsto \Theta+\mathcal{I}_{\pi}$ is a homomorphism from $3(\mathrm{~g})$ to the center $Z\left(\mathscr{D}_{\pi}\right)$ of the algebra $\mathscr{D}_{\pi}$. In general this homomorphism is neither surjective nor injective; for example, there are exactly four noncompact irreducible symmetric spaces for which it is not surjective [12]. In the case of the scalar slash actions of the real and complex Jacobi groups, we shall see that it is surjective but not injective.

Let us note that one can use (33) to prove that for any $\Theta \in 3(\mathrm{~g})$, the left action $\lambda(\Theta)$ of $\Theta$ on $C_{\text {sec }}^{\infty}\left(G \times_{K} V\right)$ is precisely $(\overline{\rho \otimes \beta})\left(\Theta^{T}+\mathcal{I}_{\pi}\right)$. (For the details, check that both are invariant differential operators and that they are equal at $\bar{e}$.)

4.2. Cocycles and slash actions. In this section we make the connection between $G$-bundles and slash actions. Given $x \in G$, denote the $\operatorname{coset} x K$ by $\bar{x}$. For any vector space $V$, a $V$-valued 1-cocycle of $G$ on $G / K$ is a smooth function $\alpha: G \times(G / K) \rightarrow$ $\mathrm{GL}(V)$ satisfying the cocycle equation

$$
\alpha(g h, \bar{x})=\alpha(h, \bar{x}) \alpha(g, h \bar{x}) .
$$

The slash action $\left.\right|_{\alpha}$ of $G$ on $C^{\infty}(G / K) \otimes V$ associated to $\alpha$ is the right action

$$
\left.f\right|_{\alpha}[g](\bar{x}):=\alpha(g, \bar{x}) f(g \bar{x}) .
$$


The corresponding left action $\lambda_{\alpha}$ is $\lambda_{\alpha}(g)(f):=\left.f\right|_{\alpha}\left[g^{-1}\right]$. We write $\mathbb{D}_{\alpha}(G / K)$ for the algebra of differential operators on $C^{\infty}(G / K) \otimes V$ invariant under $\left.\right|_{\alpha}$.

Define $\pi_{\alpha}: K \rightarrow \operatorname{GL}(V)$ by $\pi_{\alpha}(k):=\alpha(k, \bar{e})^{-1}$. By the cocycle equation, it is a representation of $K$, and so we have the $G$-bundle $G \times_{K} V$. Let us note a misprint in Lemma 4.6 of [4]: $\chi_{\alpha}(k)$ should be defined to be $\alpha(k, e K)^{-1}$.

Now check that $\tau_{\alpha}(\bar{x}, v):=[x, \alpha(x, \bar{e}) v]$ is a well-defined smooth map from $(G / K) \times V$ to $G \times_{K} V$, with inverse $\tau_{\alpha}^{-1}[x, v]=\left(\bar{x}, \alpha(x, \bar{e})^{-1} v\right)$. Use the same symbol $\tau_{\alpha}$ for the bijection from $C^{\infty}(G / K) \otimes V$ to $C_{\sec }^{\infty}\left(G \times_{K} V\right)$ defined by $\tau_{\alpha}(f)(x):=\alpha(x, \bar{e}) f(\bar{x})$ (recall that we regard $C_{\mathrm{sec}}^{\infty}\left(G \times_{K} V\right)$ as a subspace of $\left.C^{\infty}(G) \otimes V\right)$. We leave the proof of the following lemma to the reader.

Lemma 4.2. The map $\tau_{\alpha}:(G / K) \times V \rightarrow G \times_{K} V$ is a topological trivialization of $G \times_{K} V$. The map $\tau_{\alpha}: C^{\infty}(G / K) \otimes V \rightarrow C_{\mathrm{sec}}^{\infty}\left(G \times_{K} V\right)$ is an equivalence from the action $\lambda_{\alpha}$ of $G$ to the canonical action $\lambda$. This equivalence is an order 0 differential operator, and so it induces an order-preserving algebra isomorphism $T_{\alpha}$ from $\mathbb{D}_{\alpha}(G / K)$ to $\mathbb{D}\left(G \times_{K} V\right)$, defined by $T_{\alpha}(D):=\tau_{\alpha} \circ D \circ \tau_{\alpha}^{-1}$.

Define $R_{\alpha}$ to be the filtration-preserving algebra isomorphism $T_{\alpha}^{-1} \circ(\overline{\rho \otimes \beta})$ from $\mathscr{D}_{\pi}$ to $\mathbb{D}_{\alpha}(G / K)$. Given $f$ in $C^{\infty}(G / K) \otimes V$ and an element $P$ of $\varepsilon_{\pi}$ whose image $\bar{P}$ modulo $\mathcal{I}_{\pi}$ is in $\mathscr{D}_{\pi}$, unwinding the definitions yields

$$
\left(R_{\alpha}(\bar{P}) f\right)(\bar{x})=\left.\alpha(x, \bar{e})^{-1}(\rho \otimes \beta)(P)\right|_{y=x}\{y \mapsto \alpha(y, \bar{e}) f(\bar{y})\} .
$$

For completeness we briefly discuss cohomology. Fix vector spaces $V$ and $V^{\prime}$ of the same dimension, and let $\alpha$ and $\alpha^{\prime}$ be $V$ - and $V^{\prime}$-valued 1-cocycles of $G$ on $G / K$, respectively. They are said to be cohomologous if there exists a smooth function $b$ from $G / K$ to the set of invertible maps from $V$ to $V^{\prime}$, the coboundary, such that

$$
\alpha^{\prime}(g, \bar{x})=b(\bar{x}) \alpha(g, \bar{x}) b(g \bar{x})^{-1} .
$$

In this case, one checks that $f \mapsto b f$ intertwines the $G$-actions $\left.\right|_{\alpha}$ and $\left.\right|_{\alpha^{\prime}}$.

Lemma 4.3. Two cocycles $\alpha$ and $\alpha^{\prime}$ are cohomologous if and only if $\pi_{\alpha}$ and $\pi_{\alpha^{\prime}}$ are equivalent representations of $K$. If $G / K$ is simply connected, then given any representation $\pi$ of $K$ there exists a cocycle $\alpha$ such that $\pi_{\alpha}=\pi$.

Proof. If $b: G / K \rightarrow \operatorname{Hom}\left(V, V^{\prime}\right)$ is a coboundary from $\alpha$ to $\alpha^{\prime}$, then $b(\bar{e})$ is an equivalence from $\pi_{\alpha}$ to $\pi_{\alpha^{\prime}}$. Conversely, if $B: V \rightarrow V^{\prime}$ is an equivalence from $\pi_{\alpha}$ to $\pi_{\alpha^{\prime}}$, one checks that $b(\bar{x}):=\alpha^{\prime}(x, \bar{e})^{-1} B \alpha(x, \bar{e})$ is a well-defined coboundary.

If $G / K$ is simply connected, then $\pi: K \rightarrow \mathrm{GL}(V)$ extends to a smooth map $A: G \rightarrow \operatorname{GL}(V)$ such that $A(g k)=A(g) \pi(k)$ for all $g \in G$ and $k \in K$, and $\alpha(g, \bar{x}):=A(x) A(g x)^{-1}$ is a well-defined cocycle with $\pi_{\alpha}=\pi$. 
4.3. The reductive case. Throughout this section we assume that there exists a $K$-splitting $\mathfrak{k} \oplus \mathfrak{m}$ of $\mathfrak{g}$. In this case we have the $K$-injection

$$
\operatorname{Sym} \otimes 1: S(\mathfrak{m}) \otimes \operatorname{End}(V) \rightarrow \mathcal{E}_{\pi} .
$$

The image of this map is a $K$-invariant complement of $\mathcal{I}_{\pi}$ in $\mathcal{E}_{\pi}$. This leads to the following proposition, which is proven in [11]. It corresponds to Corollary 4.3 of [4].

Proposition 4.4. Suppose that $\mathfrak{\mathfrak { }} \oplus \mathfrak{m}$ is a $K$-splitting of $\mathfrak{g}$. Then under the $K$-action $\mathrm{ad} \otimes \mathrm{ad}_{\pi}, \mathcal{I}_{\pi}^{K}$ is a two-sided ideal in the algebra $\mathcal{E}_{\pi}^{K}$, and $\mathscr{D}_{\pi}$ is naturally isomorphic to $\mathcal{E}_{\pi}^{K} / \mathcal{I}_{\pi}^{K}$. Moreover, the following map is a filtration-preserving linear bijection (but in general not an algebra isomorphism except at the graded level):

$$
\delta_{\pi}:(S(\mathfrak{m}) \otimes \operatorname{End}(V))^{K} \rightarrow \mathscr{D}_{\pi}, \quad \delta_{\pi}(\Omega):=(\operatorname{Sym} \otimes 1)(\Omega)+\mathcal{I}_{\pi} .
$$

Suppose that $\gamma$ is a scalar cocycle. In (21) of [4] we gave a linear bijection $\operatorname{IDO}_{\gamma}$ from $\delta(\mathfrak{m})^{K}$ to $\mathbb{D}_{\gamma}(G / K)$ :

$$
\left(\operatorname{IDO}_{\gamma}(\Omega) f\right)(\bar{x}):=\left.\gamma^{-1}(x, \bar{e}) \lambda_{\gamma}(\operatorname{Sym}(\Omega))\right|_{\bar{y}=\bar{e}}\{\bar{y} \mapsto \gamma(x, \bar{y}) f(x \bar{y})\} .
$$

Lemma 4.5. In the setting of (37), $\operatorname{IDO}_{\gamma}(\Omega)=(-1)^{\operatorname{degree}(\Omega)} R_{\gamma}(\operatorname{Sym}(\Omega))$.

Proof. Both sides are invariant differential operators, so it suffices to prove them equal at $\bar{e}$. Here $\operatorname{End}(V)=\mathbb{C}$, so $\rho \otimes \beta$ is just $\rho$. Check that for $f \in C^{\infty}(G / K) \otimes V$,

$$
\begin{aligned}
\left(\operatorname{IDO}_{\gamma}(\Omega) f\right)(\bar{e}) & =\left.\lambda_{\gamma}(\operatorname{Sym}(\Omega))\right|_{\bar{y}=\bar{e}}\{\bar{y} \mapsto f(\bar{y})\}, \\
{\left[R_{\gamma}(\operatorname{Sym}(\Omega)) f\right](\bar{e}) } & =\left.\rho(\operatorname{Sym}(\Omega))\right|_{y=e}\{y \mapsto \gamma(y, \bar{e}) f(\bar{y})\} .
\end{aligned}
$$

Consider the two right sides. Replacing $\operatorname{Sym}(\Omega)$ by an element $g$ of $G$ in the first gives $\gamma\left(g^{-1}, \bar{e}\right) f\left(\overline{g^{-1}}\right)$, and doing so in the second gives $\gamma(g, \bar{e}) f(\bar{g})$. The lemma now follows from the same reasoning that gives (33).

In light of Lemma 4.5, we may extend (37) to define a filtration-preserving linear isomorphism for arbitrary cocycles as follows:

$$
\begin{gathered}
\mathrm{IDO}_{\alpha}:(S(\mathrm{~m}) \otimes \operatorname{End}(V))^{K} \rightarrow \mathbb{D}_{\alpha}(G / K), \\
\operatorname{IDO}_{\alpha}(\Omega):=(-1)^{\operatorname{degree}(\Omega)} R_{\alpha} \circ \delta_{\pi_{\alpha}}(\Omega) .
\end{gathered}
$$

It is an algebra isomorphism at the graded level.

Suppose that $\gamma: K \rightarrow \mathbb{C}$ is a scalar cocycle and $\alpha$ is a $V$-valued cocycle. Then $\gamma \alpha$ is a second $V$-valued cocycle and the two actions ad $\operatorname{ad}_{\pi_{\alpha}}$ and $\operatorname{ad}_{\pi_{\gamma \alpha}}$ of $K$ on $\operatorname{End}(V)$ are identical, so $\mathrm{IDO}_{\alpha}$ and $\mathrm{IDO}_{\gamma \alpha}$ have the same domain. In this case, (36) gives the following lemma. 
Lemma 4.6. In the above setting, $\operatorname{IDO}_{\alpha}(\Omega)$ and $\operatorname{IDO}_{\gamma \alpha}(\Omega)$ have the same symbol for all $\Omega$ in $(S(\mathfrak{m}) \otimes \operatorname{End}(V))^{K}$.

\section{Invariant operators in the real case}

The real Jacobi group $G^{J}(\mathbb{R}):=\mathrm{SL}_{2}(\mathbb{R}) \ltimes\left(\mathbb{R}^{2} \tilde{\times} \mathbb{R}\right)$ is obtained by taking $D$ to be $\mathbb{R}$ in Section 2.1. Recall its center $Z^{J}(\mathbb{R})$ and subgroup $K^{J}(\mathbb{R})$ :

$$
Z^{J}(\mathbb{R})=\{I\} \times\{0\} \times \mathbb{R}, \quad K^{J}(\mathbb{R}):=\mathrm{SO}_{2} \times Z^{J}(\mathbb{R}) .
$$

Let $\mathbb{H}$ denote the complex upper half plane. The formulas in Lemma 2.1 define a transitive action of $G^{J}(\mathbb{R})$ on $\mathbb{H} \times \mathbb{C}$ such that stabilizer of $(i, 0)$ is $K^{J}(\mathbb{R})$. For $k \in \mathbb{Z}$ and $m \in \mathbb{C}$, there is a well-known right action $\left.\right|_{k, m} ^{\mathbb{R}}$ of $G^{J}(\mathbb{R})$ on $C^{\infty}(\mathbb{H} \times \mathbb{C})$ [7]. Let us denote the algebra of $\left.\right|_{k, m} ^{\mathbb{R}}$-invariant differential operators by $\mathbb{D}_{k, m}^{\mathbb{R}}$. In this section we apply Section 4 to obtain generators and relations for $\mathbb{D}_{k, m}^{\mathbb{R}}$. This requires only Helgason's results [11], as Proposition 4.4 applies.

The algebra $\mathbb{D}_{k, m}^{\mathbb{R}}$ is not directly related to the number theoretic part of this paper, but we have two reasons for including it. First, many of the results we obtain en route are useful in the complex case. Second, although generators and relations for the graded commutative symbol algebra of $\mathbb{D}_{k, m}^{\mathbb{R}}$ were obtained in [1], to our knowledge the relations in $\mathbb{D}_{k, m}^{\mathbb{R}}$ itself have not yet appeared. We will use them to prove that the center of $\mathbb{D}_{k, m}^{\mathbb{R}}$ is precisely the action of $3\left(\mathfrak{g}^{J}(\mathbb{R})\right)$ under $\left.\right|_{k, m} ^{\mathbb{R}}$, and that it is generated by a single cubic operator, the action of the "cubic Casimir element" of $G^{J}(\mathbb{R})$. This element is called $C$ on page 38 of [1], and its action is called $\ell^{k, m}$ in [17].

Let us remark that $\left.\right|_{k, m} ^{\mathbb{R}}$ differs from the complex right action $\left.\right|_{k, m}$ defined in Section 2.1 not only in that $k$ must be integral, but also in that varying $k$ does not produce equivalent actions. Indeed, in the complex case the family $\left\{\left.\right|_{0, m}: m \in \mathbb{C}\right\}$ classifies all scalar right actions such that the action of the center of the group is bounded, while in the real case $\left\{\left.\right|_{k, m} ^{\mathbb{R}}: k \in \mathbb{Z}, m \in \mathbb{R}\right\}$ does so; see Lemma 4.3.

We will not need the explicit formula for $\left.\right|_{k, m} ^{\mathbb{R}}$; only the associated characters of $K$. However, we give it for reference. Define $a^{\mathbb{R}}: G^{J}(\mathbb{R}) \times(\mathbb{H} \times \mathbb{C}) \rightarrow \mathbb{C}$ by

$$
a^{\mathbb{R}}((M, X, \kappa),(\tau, z)):=\kappa+\lambda \mu+2 \lambda z+\lambda^{2} \tau-\gamma(z+\lambda \tau+\mu)^{2}(\gamma \tau+\delta)^{-1}
$$

(see (3)). For $k \in \mathbb{Z}$ and $m \in \mathbb{C}$, define $\alpha_{k, m}^{\mathbb{R}}: G^{J}(\mathbb{R}) \times(\mathbb{H} \times \mathbb{C}) \rightarrow \mathbb{C}^{\times}$by

$$
\alpha_{k, m}^{\mathbb{R}}((M, X, \kappa),(\tau, z)):=(\gamma \tau+\delta)^{-k} \exp \left\{2 \pi i m a^{\mathbb{R}}((M, X, \kappa),(\tau, z))\right\} .
$$

As in the complex case (see Proposition 2.2), $\alpha_{k, m}^{\mathbb{R}}$ is a cocycle of the action of $G^{J}(\mathbb{R})$ on $\mathbb{H} \times \mathbb{C}$ [7]. The associated slash action of $G^{J}(\mathbb{R})$ on $C^{\infty}(\mathbb{H} \times \mathbb{C})$ is

$$
\left(\left.f\right|_{k, m}(M, X, \kappa)\right)(\tau, z):=\alpha_{k, m}^{\mathbb{R}}((M, X, \kappa),(\tau, z)) f((M, X, \kappa) \circ(\tau, z)) .
$$


5.1. The real Jacobi Lie algebra. Recall from Section 4 that the Lie algebra $g^{J}(\mathbb{R})$ of $G^{J}(\mathbb{R})$ is defined to be the complexification of the real Lie algebra $\mathrm{g}_{0}^{J}(\mathbb{R})$. In order to proceed it is necessary to reproduce some data from Section 5.2 of [4]:

$$
\mathfrak{g}^{J}(\mathbb{R})=\left\{(M, X, \kappa): M \in \mathfrak{s l}_{2} \mathbb{C}, X \in \mathbb{C}^{2}, \kappa \in \mathbb{C}\right\},
$$

with Lie bracket

$$
\left[(M, X, \kappa),\left(M^{\prime}, X^{\prime}, \kappa^{\prime}\right)\right]=\left(\left[M, M^{\prime}\right], X M^{\prime}-X^{\prime} M, 2 \operatorname{det}\left(\begin{array}{c}
X \\
X^{\prime}
\end{array}\right)\right) .
$$

Setting $J=\left(\begin{array}{cc}0 & -1 \\ 1 & 0\end{array}\right)$ and $\left.h(z)=z^{-2}\left(e^{z}-z-1\right)\right)$, the exponential map is

$$
\exp (M, X, \kappa)=\left(e^{M}, X\left(\frac{e^{M}-I}{M}\right), \kappa-X h(M) J X^{t}\right) .
$$

The standard basis $\{H, E, F, Z, e, f\}$ of $\mathrm{g}^{J}(\mathbb{R})$ is defined by

$$
\begin{aligned}
& H:=\left(\begin{array}{cc}
1 & 0 \\
0 & -1
\end{array}\right), \quad E:=\left(\begin{array}{ll}
0 & 1 \\
0 & 0
\end{array}\right), \quad F:=\left(\begin{array}{ll}
0 & 0 \\
1 & 0
\end{array}\right), \\
& Z:=(0,(0,0), 1), \quad e:=(0,(0,1), 0), \quad f:=(0,(1,0), 0) .
\end{aligned}
$$

The standard basis is not compatible with $K^{J}(\mathbb{R})$, so we define the "tilde" basis:

$$
\begin{aligned}
& \tilde{H}:=i(F-E), \quad \widetilde{E}:=\frac{1}{2}(H+i(F+E)), \quad \tilde{F}:=\frac{1}{2}(H-i(F+E)), \\
& \tilde{Z}:=\frac{1}{2} i Z, \quad \tilde{e}:=\frac{1}{2}(f+i e), \quad \tilde{f}:=\frac{1}{2}(f-i e) .
\end{aligned}
$$

Observe that while the standard basis is both a real basis of $g_{0}^{J}(\mathbb{R})$ and a complex basis of $\mathrm{g}^{J}(\mathbb{R})$, the tilde basis is only the latter. The brackets in the tilde basis are identical to those in the standard basis: in other words, $\tau(B)=\widetilde{B}$ for $B \in\{H, E, F, Z, e, f\}$ defines an automorphism of $\mathrm{g}^{J}(\mathbb{R})$. In fact, writing $\zeta$ for $\exp \{\pi i / 4\}$ and $T$ for the order 6 element $\frac{1}{\sqrt{2}}\left(\begin{array}{cc}\zeta & \zeta \\ \zeta^{3} & \zeta^{-1}\end{array}\right)$ of $\mathrm{SU}_{2}, \tau$ is conjugation by $(T, 0,0)$ followed by the $\mathrm{SL}_{2}$-automorphism of the Heisenberg algebra which multiplies $e$ and $f$ by $\zeta / \sqrt{2}$ and $Z$ by $i / 2$.

There is a unique $K^{J}(\mathbb{R})$-splitting $\mathfrak{k}^{J}(\mathbb{R}) \oplus \mathfrak{m}^{J}(\mathbb{R})$ of $\mathfrak{g}^{J}(\mathbb{R})$ :

$$
\mathfrak{k}^{J}(\mathbb{R})=\operatorname{Span}_{\mathbb{C}}\{\tilde{H}, \tilde{Z}\}, \quad \mathfrak{m}^{J}(\mathbb{R}):=\operatorname{Span}_{\mathbb{C}}\{\tilde{E}, \tilde{F}, \tilde{e}, \tilde{f}\} .
$$

We will need the following easy lemma. A proof may be found in [1].

Lemma 5.1. The commutative algebra $S\left(\mathfrak{m}^{J}(\mathbb{R})\right)^{K^{J}(\mathbb{R})}$ has basis $\left\{\widetilde{E}^{i_{E}} \tilde{F}^{i_{F}} \tilde{e}^{i_{e}} \tilde{f}^{i_{f}}\right.$ : $\left.2 i_{E}+i_{e}=2 i_{F}+i_{f}\right\}$. It is generated by $\tilde{F} \tilde{E}$, $\tilde{f} \tilde{e}, \tilde{F} \tilde{e}^{2}$, and $\tilde{f}^{2} \tilde{E}$. The relation ideal for these generators is generated by $(\tilde{F} \tilde{E})(\tilde{f} \tilde{e})^{2}=\left(\tilde{F} \tilde{e}^{2}\right)\left(\tilde{f}^{2} \tilde{E}\right)$. 
There is a "Cartan automorphism" $\theta$ of $g^{J}(\mathbb{R})$, defined as follows: it negates $H$, exchanges $E$ and $-F$, fixes $Z$, and sends $e$ to $f$ to $-e$. Note that it is of order 4 . The tilde Cartan automorphism $\tilde{\theta}:=\tau \circ \theta \circ \tau^{-1}$ will be a useful tool.

Definition 5. Let $C$ be the following element of $\mathcal{U}\left(g^{J}(\mathbb{R})\right)$ :

$$
C:=f^{2} E-F e^{2}-(H+2) f e+(H+1)(H+2) Z+4 F E Z .
$$

Proposition 5.2. $3\left(\mathrm{~g}^{J}(\mathbb{R})\right)$ is the polynomial algebra $\mathbb{C}[Z, C]$. We have $C^{T}=-C$, $\theta(C)=C$, and $\tau(C)=\frac{i}{2} C$.

Proof. In this proof we write $\mathfrak{g}$ for $\mathfrak{g}^{J}(\mathbb{R})$. Since Sym: $\delta(\mathfrak{g})^{\mathfrak{g}} \rightarrow 3(\mathfrak{g})$ is a linear bijection, we must compute $S(\mathfrak{g})^{\mathfrak{g}}$. First we compute $S(\mathfrak{g})^{\mathfrak{s l}_{2} \mathbb{C}}$.

Up to equivalence, $\mathfrak{s l}_{2} \mathbb{C}$ has a unique irreducible $n+1$-dimensional representation $L_{n}$ for all $n \geq 0$. The following formulas are classical:

$$
\begin{gathered}
S^{n}\left(L_{1}\right) \cong L_{n}, \quad S^{n}\left(L_{2}\right) \cong L_{2 n} \oplus L_{2 n-4} \oplus \cdots \oplus L_{2 \operatorname{Res}_{2}(n)}, \\
L_{n} \otimes L_{m} \cong L_{n+m} \oplus L_{n+m-2} \oplus \cdots \oplus L_{|n-m|},
\end{gathered}
$$

where $\cong$ denotes $\mathfrak{s}_{2} \mathbb{C}$-equivalence and $\operatorname{Res}_{2}$ denotes the residue modulo 2 .

Under its subalgebra $\mathfrak{s} \mathfrak{l}_{2} \mathbb{C}, \mathfrak{g}$ is equivalent to $L_{0} \oplus L_{1} \oplus L_{2}$ : the copy of $L_{0}$ is $\mathbb{C} Z$, the copy of $L_{1}$ is $\operatorname{Span}\{e, f\}$, and the copy of $L_{2}$ is $\mathfrak{s} \mathfrak{l}_{2} \mathbb{C}$ itself. By (44), both $S^{2}\left(L_{2}\right)$ and $L_{2} \otimes S^{2}\left(L_{1}\right)$ contain $s \mathfrak{l}_{2} \mathbb{C}$-invariants, unique up to a scalar. They are

$$
P_{2}:=4 F E+H^{2}, \quad P_{3}:=f^{2} E-F e^{2}-H f e .
$$

A consideration of $L_{0^{-}}, L_{1^{-}}$, and $L_{2}$-degrees shows that the $\mathfrak{s} \mathfrak{l}_{2} \mathbb{C}$-invariants $Z$, $P_{2}$, and $P_{3}$ in the commutative algebra $\delta(g)$ are algebraically independent. Now $S\left(L_{1} \oplus L_{2}\right)=\bigoplus_{i, j} S^{i}\left(L_{1}\right) \otimes S^{j}\left(L_{2}\right)$, and by $(44)$, the $(i, j)^{\text {th }}$ summand on the right has no invariants unless $2 j-i=4 n$ for some $n \in \mathbb{N}$, when it has up to a scalar one. Since $P_{2}^{n} P_{3}^{j-2 n}$ is such an invariant, we find that $S\left(L_{1} \oplus L_{2}\right)^{\mathfrak{N} \mathfrak{N}_{2} \mathbb{C}}=\mathbb{C}\left[P_{2}, P_{3}\right]$. This leads easily to the fact that $S(g)^{\mathfrak{s l}_{2} \mathbb{C}}$ is the polynomial algebra $\mathbb{C}\left[Z, P_{2}, P_{3}\right]$.

Since $\mathfrak{s l}_{2} \mathbb{C}$ and $e$ generate $\mathfrak{g}, S(\mathfrak{g})^{\mathfrak{g}}$ is the set of $\operatorname{ad}(e)$-invariants in $\mathbb{C}\left[Z, P_{2}, P_{3}\right]$. Direct computation shows that $\operatorname{ad}(e)\left(P_{3}\right)=-Z \operatorname{ad}(e)\left(P_{2}\right) \neq 0$. Since $\left\{Z, P_{2}, P_{3}+\right.$ $\left.P_{2} Z\right\}$ is an algebraic basis of $\mathbb{C}\left[Z, P_{2}, P_{3}\right]$, we find that $S(\mathrm{~g})^{\mathrm{g}}$ is $\mathbb{C}\left[Z, P_{3}+P_{2} Z\right]$. To finish, verify $\operatorname{Sym}\left(P_{3}+P_{2} Z\right)=C-Z, \theta\left(P_{2}\right)=\tau\left(P_{2}\right)=P_{2}$, and $\theta\left(P_{3}\right)=$ $-2 i \tau\left(P_{3}\right)=P_{3}$.

5.2. The algebra $\mathbb{D}_{\boldsymbol{k}, \boldsymbol{m}}^{\mathbb{R}}$. By definition, $\mathbb{D}_{k, m}^{\mathbb{R}}$ is $\mathbb{D}_{\alpha_{k, m}^{\mathbb{R}}}\left(G^{J}(\mathbb{R}) / K^{J}(\mathbb{R})\right)$. We maintain this pattern of abbreviation: write $\lambda_{k, m}^{\mathbb{R}}$ for the left action corresponding to $\left.\right|_{k, m} ^{\mathbb{R}}$, $\pi_{k, m}^{\mathbb{R}}$ for the character $\pi_{\alpha_{k, m}^{\mathbb{R}}}$ of $K^{J}(\mathbb{R})$ associated to it by Section 4.2 , and $\mathcal{E}_{k, m}^{\mathbb{R}}$, $\mathcal{I}_{k, m}^{\mathbb{R}}$, and $\mathscr{D}_{k, m}^{\mathbb{R}}$ for the corresponding spaces defined by (34). Similarly, we have the 
filtration-preserving algebra isomorphism $R_{k, m}^{\mathbb{R}}: \mathcal{D}_{k, m}^{\mathbb{R}} \rightarrow \mathbb{D}_{k, m}^{\mathbb{R}}$ from (36), and the linear bijections from Section 4.3:

$$
\delta_{k, m}^{\mathbb{R}}: S\left(\mathfrak{m}^{J}(\mathbb{R})\right)^{\mathfrak{k}^{J}(\mathbb{R})} \rightarrow \mathcal{D}_{k, m}^{\mathbb{R}}, \quad \operatorname{IDO}_{k, m}^{\mathbb{R}}=R_{k, m}^{\mathbb{R}} \circ \delta_{k, m}^{\mathbb{R}} \circ(-1)^{\text {degree }} .
$$

Here $\mathcal{E}_{k, m}^{\mathbb{R}}$ is simply $\mathcal{U}\left(\mathfrak{g}^{J}(\mathbb{R})\right)$, and the reader may check that $\pi_{k, m}^{\mathbb{R}}$ maps $\tilde{H}$ to $-k$ and $\widetilde{Z}$ to $\pi m$, so $\mathcal{I}_{k, m}^{\mathbb{R}}$ is the left ideal generated by $\tilde{H}-k$ and $\widetilde{Z}+\pi m$.

We now define several elements $\delta_{\bullet}(\mathbb{R}, k, m)$ (we usually write simply $\delta_{\bullet}$ ) of $\mathbb{D}_{k, m}^{\mathbb{R}}$ :

$$
\begin{gathered}
\delta_{z}:=\delta_{k, m}^{\mathbb{R}}(\tilde{f} \tilde{e}), \quad \delta_{\tau}:=\delta_{k, m}^{\mathbb{R}}(\tilde{F} \tilde{E}), \quad \delta_{f}:=\delta_{k, m}^{\mathbb{R}}\left(\tilde{f}^{2} \tilde{E}\right), \quad \delta_{e}:=\delta_{k, m}^{\mathbb{R}}\left(\tilde{F} \tilde{e}^{2}\right), \\
\delta_{b}:=\delta_{f}+\delta_{e}, \quad \delta_{c}:=\delta_{f}-\delta_{e}-4 \pi m \delta_{\tau}-k \delta_{z} .
\end{gathered}
$$

Note that the automorphism $\tilde{\theta}$ of $\mathrm{g}^{J}(\mathbb{R})$ induces an algebra isomorphism $\tilde{\theta}_{k, m}$ from $\mathbb{D}_{k, m}^{\mathbb{R}}$ to $\mathscr{D}_{-k, m}^{\mathbb{R}}$. This isomorphism is

$$
\delta_{z} \mapsto-\delta_{z}, \quad \delta_{\tau} \mapsto \delta_{\tau}, \quad \delta_{f} \mapsto-\delta_{e}, \quad \delta_{e} \mapsto-\delta_{f}, \quad \delta_{b} \mapsto-\delta_{b}, \quad \delta_{c} \mapsto \delta_{c} .
$$

Theorem 5.3. The algebra $\mathcal{D}_{k, m}^{\mathbb{R}}$ has basis $\left\{\delta_{\tau}^{i_{\tau}} \delta_{b}^{i_{b}} \delta_{z}^{i_{z}} \delta_{c}^{i_{c}}: i_{\tau}, i_{z}, i_{c} \in \mathbb{N}, i_{b}=0,1\right\}$. Its center is $\mathbb{C}\left[\delta_{c}\right]$. In it, $C+\mathcal{I}_{k, m}^{\mathbb{R}}=-2 i\left(\delta_{c}-\pi m\left(k^{2}+\frac{2}{3}\right)\right)$. It has relations $\left[\delta_{z}, \delta_{\tau}\right]=\delta_{b}$,

$$
\begin{aligned}
{\left[\delta_{z}, \delta_{b}\right]=} & 2 \delta_{z}^{2}+4 \pi m \delta_{c}+16 \pi^{2} m^{2} \delta_{\tau}+4 \pi m k \delta_{z}+\frac{10}{3} \pi^{2} m^{2}, \\
{\left[\delta_{\tau}, \delta_{b}\right]=} & -4 \delta_{\tau} \delta_{z}-2 \delta_{b}-k \delta_{c}-4 \pi m k \delta_{\tau}-k^{2} \delta_{z}+\frac{2}{3} \pi m k, \\
\delta_{b}^{2}= & \left(\delta_{c}+4 \pi m \delta_{\tau}+k \delta_{z}\right)^{2}+4 \delta_{\tau} \delta_{z}^{2}+4 \delta_{b} \delta_{z} \\
& \quad+\frac{20}{3} \pi m \delta_{c}+\frac{116}{3} \pi^{2} m^{2} \delta_{\tau}-\frac{4}{3} \pi m k \delta_{z}+\frac{64}{9} \pi^{2} m^{2} .
\end{aligned}
$$

Proof. The first sentence was proven in [1], and it follows from Lemma 5.1 and the fact that $\delta_{k, m}^{\mathbb{R}}$ is an algebra isomorphism at the graded level. For the relations, we compute in $\mathscr{D}_{k, m}^{\mathbb{R}}$ as prescribed by [11]: using the relations of $\mathcal{U}\left(\mathrm{g}^{J}(\mathbb{R})\right)$, move all $\tilde{H}$ 's and $\widetilde{Z}$ 's to the right and then replace them with $k$ and $-\pi m$, respectively. Deduce the following equations in $\mathscr{D}_{k, m}^{\mathbb{R}}$ :

$$
\begin{gathered}
\tilde{f} \tilde{e}=\delta_{z}-\pi m, \quad \tilde{e} \tilde{f}=\delta_{z}+\pi m, \\
\tilde{F} \tilde{E}=\delta_{\tau}-\frac{1}{2} k, \quad \tilde{E} \tilde{F}=\delta_{\tau}+\frac{1}{2} k, \\
\tilde{f}^{2} \tilde{E}=\delta_{f}+\delta_{z}-\frac{1}{3} \pi m, \quad \tilde{f} \tilde{E} \tilde{f}=\delta_{f}+\frac{2}{3} \pi m, \quad \tilde{E} \tilde{f}^{2}=\delta_{f}-\delta_{z}-\frac{1}{3} \pi m, \\
\tilde{F} \tilde{e}^{2}=\delta_{e}-\delta_{z}+\frac{1}{3} \pi m, \quad \tilde{e} \tilde{F} \tilde{e}=\delta_{e}-\frac{2}{3} \pi m, \quad \tilde{e}^{2} \tilde{F}=\delta_{e}+\delta_{z}+\frac{1}{3} \pi m .
\end{gathered}
$$


In fact, it is only necessary to verify half of these equations; the other half then follow by applying $\tilde{\theta}_{k, m}$ and negating $k$. Using them, we compute $\left[\delta_{z}, \delta_{\tau}\right]=$ $[\tilde{f} \tilde{e}, \widetilde{F} \widetilde{E}]=\tilde{f}^{2} \widetilde{E}+\widetilde{F} \tilde{e}^{2}=\delta_{b}$. Similar computations (again using $\tilde{\theta}_{k, m}$ to halve the work) yield

$$
\left[\delta_{z}, \delta_{f}\right]=\delta_{z}^{2}+4 \pi m \delta_{f}+\frac{5}{3} \pi^{2} m^{2}, \quad\left[\delta_{z}, \delta_{e}\right]=\delta_{z}^{2}-4 \pi m \delta_{e}+\frac{5}{3} \pi^{2} m^{2},
$$

which proves the $\left[\delta_{z}, \delta_{b}\right]$ relation. Next, prove the $\left[\delta_{\tau}, \delta_{b}\right]$ relation by verifying $\left[\delta_{\tau}, \delta_{f}\right]=-2 \delta_{\tau} \delta_{z}-\delta_{b}-k \delta_{f}+\frac{1}{3} \pi m k, \quad\left[\delta_{\tau}, \delta_{e}\right]=-2 \delta_{\tau} \delta_{z}-\delta_{b}+k \delta_{e}+\frac{1}{3} \pi m k$.

For the $\delta_{b}^{2}$ relation, set $L=\delta_{b}^{2}-\left(\delta_{c}+4 \pi m \delta_{\tau}+k \delta_{z}\right)^{2}$. Check $L=2\left(\delta_{f} \delta_{e}+\delta_{e} \delta_{f}\right)$. Use $\delta_{f}=\tilde{f} \tilde{E} \tilde{f}-\frac{2}{3} \pi m$ and $\delta_{e}=\tilde{e} \tilde{F} \tilde{e}+\frac{2}{3} \pi m$ to obtain

$$
L=2(\tilde{f} \tilde{E} \tilde{f} \tilde{e} \tilde{F} \tilde{e}+\tilde{e} \tilde{F} \tilde{e} \tilde{f} \tilde{E} \tilde{f})+\frac{8}{3} \pi m(\tilde{f} \tilde{E} \tilde{f}-\tilde{e} \tilde{F} \tilde{e})-\frac{16}{9} \pi^{2} m^{2} .
$$

Now set $S=\tilde{f} \tilde{E} \tilde{f} \tilde{e} \tilde{F} \tilde{e}$. Commute $\tilde{e} \tilde{F}$ to get $S=(\tilde{f} \tilde{E} \tilde{f})(\tilde{f} \tilde{e})+\tilde{f} \tilde{E} \tilde{F} \tilde{f} \tilde{e}^{2}$. Commute $\tilde{f} \widetilde{E}$ in the second summand and the $\tilde{e} \tilde{f}$ where appropriate to arrive at

$$
S=\delta_{b}\left(\delta_{z}-\pi m\right)+\left(\delta_{\tau}+\frac{1}{2} k\right)\left(\delta_{z}-\pi m\right)\left(\delta_{z}-3 \pi m\right)-2 \pi m\left(\delta_{e}-\frac{2}{3} \pi m\right) .
$$

Note that $\tilde{e} \tilde{F} \tilde{e} \tilde{f} \tilde{E} \tilde{f}$ is $\tilde{\theta}_{k, m}(\tilde{f} \tilde{E} \tilde{f} \tilde{e} \tilde{F} \tilde{e})$ with $k$ negated. Using these facts to simplify $L$ gives the relation.

The formula for the image of $C$ follows easily from our collection of equations, and it shows that $\delta_{c}$ is indeed central. In order to prove that it generates $Z\left(D_{k, m}^{\mathbb{R}}\right)$, suppose first that $\epsilon$ is an element of $\mathfrak{D}_{k, m}^{\mathbb{R}}$ of order $n$ which commutes with $\delta_{z}$. In light of the basis of $\mathscr{D}_{k, m}^{\mathbb{R}}$, it has a unique expression as $\sum_{i} \delta_{\tau}^{i}\left(a_{i}+\delta_{b} b_{i}\right)$, where $a_{i}$ and $b_{i}$ are in $\mathbb{C}\left[\delta_{z}, \delta_{c}\right]$.

Note that the symbols of the relations in Theorem 5.3 are independent of $k$ and $m$, as forced by Lemma 4.6. Writing $\equiv$ for equality at the symbol level,

$$
\left[\delta_{z}, \delta_{\tau}\right] \equiv \delta_{b}, \quad\left[\delta_{z}, \delta_{b}\right] \equiv 2 \delta_{z}^{2}, \quad\left[\delta_{\tau}, \delta_{b}\right] \equiv-4 \delta_{\tau} \delta_{z}, \quad \delta_{b}^{2} \equiv \delta_{c}^{2}+4 \delta_{\tau} \delta_{z}^{2} .
$$

Since symbols commute, this leads to the following equation at the $(n+1)$-symbol level:

$$
0=\left[\delta_{z}, \epsilon\right] \equiv \sum_{i} \delta_{\tau}^{i}\left((i+1) \delta_{c}^{2} b_{i+1}+2(2 i+1) \delta_{z}^{2} b_{i}+(i+1) \delta_{b} a_{i+1}\right) .
$$

Again using the basis of $\mathscr{D}_{k, m}^{\mathbb{R}}$, this yields $\epsilon \equiv a_{0}$, and so induction on $n$ shows that the commutant of $\delta_{z}$ is $\mathbb{C}\left[\delta_{z}, \delta_{c}\right]$. A similar computation shows that the commutant of $\delta_{\tau}$ in $\mathbb{C}\left[\delta_{z}, \delta_{c}\right]$ is $\mathbb{C}\left[\delta_{c}\right]$, completing the proof. 
5.3. Remarks. On page 38 of [1] an operator $C$ is introduced; we shall call it $C_{\mathrm{BS}}$. It is a Casimir operator in the sense that it acts by scalars on those irreducible representations of $G^{J}(\mathbb{R})$ on which $Z$ acts by a non-zero scalar. It is used in [1] to define Maass-Jacobi forms of $G^{J}(\mathbb{R})$, and the investigation of such forms carried out in [17] inspired the present article. Here we relate $C_{\mathrm{BS}}$ to our Casimir element $C$. We shall state several results without proof: they can all be verified by means of straightforward calculation.

The following construction is attributed by [1] to [3]. Write $\mathfrak{n}^{J}(\mathbb{R})$ for the nilpotent Heisenberg subalgebra $\operatorname{Span}\{Z, e, f\}$ of $\mathfrak{g}^{J}(\mathbb{R})$. Let $\mathcal{U}\left(\mathfrak{g}^{J}(\mathbb{R})\right)\left[Z^{-1}\right]$ be the extension of $\mathfrak{U}\left(\mathfrak{g}^{J}(\mathbb{R})\right)$ by the fraction field $\mathbb{C}(Z)$. Working in this algebra, define

$$
H^{\mathfrak{n}}:=(e f+f e) / 4 Z=(f e-Z) / 2 Z, \quad E^{\mathfrak{n}}:=e^{2} / 4 Z, \quad F^{\mathfrak{n}}:=-f^{2} / 4 Z .
$$

Lemma 5.4. The map $A \mapsto A^{\mathfrak{n}}$ for $A \in\{H, E, F\}$ defines a Lie algebra injection $\eta$ from $\mathfrak{s} \mathfrak{l}_{2}$ to $\mathcal{U}\left(\mathfrak{g}^{J}(\mathbb{R})\right)\left[Z^{-1}\right]$. It commutes with $\theta, \tau$, and the adjoint action of $\mathrm{g}^{J}(\mathbb{R})$.

For $A \in \mathfrak{s l}_{2}$, set $A^{\prime}:=A-\eta(A)$. Write $s l_{2}^{\prime}$ for $\operatorname{Span}\left\{H^{\prime}, E^{\prime}, F^{\prime}\right\}$.

Lemma 5.5. The map $A \mapsto A^{\prime}$ is a Lie algebra injection from $\mathfrak{s} \mathfrak{l}_{2}$ to $\mathfrak{U}\left(\mathfrak{g}^{J}(\mathbb{R})\right)$. Its image $\mathfrak{s l}_{2}^{\prime}$ commutes with $\mathfrak{n}^{J}(\mathbb{R})$, and so we have the algebra decomposition

$$
\mathfrak{U}\left(\mathfrak{g}^{J}(\mathbb{R})\right)\left[Z^{-1}\right]=\mathfrak{U}\left(\mathfrak{s} \mathfrak{l}_{2}^{\prime}\right) \otimes_{\mathbb{C}[Z]} \mathfrak{U}\left(\mathfrak{n}^{J}(\mathbb{R})\right)\left[Z^{-1}\right] .
$$

The Casimir element of $\mathfrak{s l}_{2}$ is $C_{\mathfrak{s} \gamma_{2}}:=\operatorname{Sym}\left(P_{2}\right)=H^{2}+2 H+4 F E$ (see (45)). It is fixed by $\theta, \tau$, and the transpose $T$, and it is classical that $3\left(\mathfrak{s I}_{2}\right)=\mathbb{C}\left[C_{\mathfrak{s}}\right]$. The Casimir elements of $\eta\left(\mathfrak{s} \mathfrak{l}_{2}\right)$ and $\mathfrak{s l} \mathfrak{l}_{2}^{\prime}$ are

$$
C_{\mathfrak{S l}_{2}}^{\mathfrak{n}}:=\left(H^{\mathfrak{n}}\right)^{2}+2 H^{\mathfrak{n}}+4 F^{\mathfrak{n}} E^{\mathfrak{n}}, \quad C_{\mathfrak{s} \mathfrak{l}_{2}}^{\prime}:=\left(H^{\prime}\right)^{2}+2 H^{\prime}+4 F^{\prime} E^{\prime} .
$$

By Lemmas 5.4 and 5.5, both are fixed by $\theta, \tau$, and $T$, and $C_{\mathfrak{s l}_{2}}^{\prime}$ is central in $\mathcal{U}\left(\mathfrak{g}^{J}(\mathbb{R})\right)\left[Z^{-1}\right]$. Therefore by Proposition 5.2, $C_{\mathfrak{s} \mathfrak{H}_{2}}^{\prime} \in \mathbb{C}(Z)[C]$. More precisely:

Lemma 5.6. $C_{\mathfrak{s l}_{2}}^{\mathfrak{n}}=-\frac{3}{4}$, and $C_{\mathfrak{s l}_{2}}^{\prime}=C Z^{-1}-\frac{3}{4}$.

Let us now connect our notation to that of [1], which is also used in [17]. The map from our standard basis of $g^{J}(\mathbb{R})$ to the one given on page 8 of [1] is

$$
H \mapsto H, \quad E \mapsto F, \quad F \mapsto G, \quad Z \mapsto R, \quad e \mapsto Q, \quad f \mapsto P .
$$

The map from our tilde basis to the one given on page 12 of [1] is

$$
\tilde{H} \mapsto Z, \quad \tilde{E} \mapsto X_{+}, \quad \tilde{F} \mapsto X_{-}, \quad \tilde{Z} \mapsto-\frac{1}{2} Z_{0}, \quad \tilde{e} \mapsto Y_{+}, \quad \tilde{f} \mapsto Y_{-} .
$$

(Their $Z$ is not the same as ours, and their $Z_{0}$ is equal to their $-i R$.) 
Their $\left.\right|_{k, m}$ is our $\left.\right|_{k, m} ^{\mathbb{R}}$. In particular, we both have $\lambda_{k, m}^{\mathbb{R}}(\widetilde{Z})=-\pi m$ (they sometimes write $\mu$ for $2 \pi m$ ). Their heat operators $D_{ \pm}$(see pages 33-38 of [1]) are elements of $\mathfrak{s} \mathfrak{l}_{2}^{\prime}$ specialized at this value of $\widetilde{Z}$ (for $m \neq 0$ ):

$$
D_{+}:=\left.\tau\left(E^{\prime}\right)\right|_{\tilde{Z}=-\pi m}, \quad D_{-}:=\left.\tau\left(F^{\prime}\right)\right|_{\tilde{Z}=-\pi m}, \quad \Delta_{1}:=\left.\tau\left(H^{\prime}\right)\right|_{\tilde{Z}=-\pi m} .
$$

The operator $C_{\mathrm{BS}}$ is $\left.\frac{1}{2} C_{\mathfrak{s} \gamma_{2}}^{\prime}\right|_{\tilde{Z}=-\pi m}$, i.e., $\frac{C}{4 i \pi m}-\frac{3}{8}$. The operator $\bigodot^{k, m}$ defined in [17] is $\lambda_{k, m}^{\mathbb{R}}\left(\frac{1}{2} C_{\mathfrak{s} \gamma_{2}}^{\prime}\right)$. By Theorem 5.3 and Lemma 5.6, it generates the center of $\mathbb{D}_{k, m}^{\mathbb{R}}$. Note that both operators are in the image of $3\left(\mathrm{~g}^{J}(\mathbb{R})\right)\left[Z^{-1}\right]$.

\section{Invariant operators in the complex case}

Recall from Section 2.1 the complex Jacobi group $G^{J}(\mathbb{C})$ and its actions $\left.\right|_{k, m}$ on $C^{\infty}\left(\mathbb{H}_{\mathcal{Q}} \times \mathcal{Q}\right)$ given by the cocycles $\alpha_{k, m}$. Although $G^{J}(\mathbb{C})$ is holomorphic, $\mathbb{H}_{\mathcal{Q}} \times \mathbb{Q}$ is not, and so as discussed in [4] we must forget the complex structure of $G^{J}(\mathbb{C})$ and regard it as a real group. As such, its real Lie algebra $g_{0}^{J}(\mathbb{C})$ is precisely the complexified Lie algebra $g^{J}(\mathbb{R})$ of the real Jacobi group $G^{J}(\mathbb{R})$ given in (39), with bracket and exponential map given by (40) and (41). This Lie algebra is 12 dimensional over $\mathbb{R}$, with standard real basis $\{B, i B: B=H, E, F, Z, e, f\}$ (see (42)).

From Lemma $2.1, G^{J}(\mathbb{C})$ acts transitively on $\mathbb{H}_{\mathcal{Q}} \times \mathcal{Q}$, and the stabilizer of $(\mathbf{k}, 0)$ is $K^{J}(\mathbb{C})=\mathrm{SU}_{2} \times \mathbb{C}$. Its real Lie algebra is

$$
\mathfrak{f}_{0}^{J}(\mathbb{C})=\operatorname{Span}_{\mathbb{R}}\{i H, F-E, i F+i E, Z, i Z\} .
$$

There is more than one $K^{J}(\mathbb{C})$-invariant splitting of $\mathfrak{g}_{0}^{J}(\mathbb{C})$. We shall use the following $K^{J}(\mathbb{C})$-invariant complement of $\mathfrak{E}_{0}^{J}(\mathbb{C})$ :

$$
\mathfrak{m}_{0}^{J}(\mathbb{C})=\operatorname{Span}_{\mathbb{R}}\{H, i F-i E, F+E, e, i e, f, \text { if }\} .
$$

6.1. Real representations of complex groups. At this point we must discuss complexifications. We will need two copies $\mathbb{C}_{i}$ and $\mathbb{C}_{j}$ of $\mathbb{C}$, with copies $i$ and $j$ of $\sqrt{-1}$, respectively. All proofs in this section will omitted.

Suppose that $U_{0}$ is a vector space over $\mathbb{C}_{i}$. Regarded as a real vector space, its complexification is $U:=U_{0} \otimes_{\mathbb{R}} \mathbb{C}_{j}$, a module over the commutative ring $\mathbb{C}_{i} \otimes_{\mathbb{R}} \mathbb{C}_{j}$. Define elements $\iota_{ \pm}:=(1 \mp j i) / 2$ of this ring, and note

$$
\iota_{ \pm}^{2}=\iota_{ \pm}, \quad \iota_{+} \iota_{-}=0, \quad \iota_{+}+\iota_{-}=1, \quad i \iota_{ \pm}= \pm j \iota_{ \pm} .
$$

Lemma 6.1. In the above setting, define $U_{ \pm}:=\iota_{ \pm}\left(U_{0}\right)$. Then $U_{ \pm}$are $\mathbb{C}_{j}$-subspaces of $U$, and $U=U_{+} \oplus U_{-}$. Regarding $U_{0}$ as complex over $\mathbb{C}_{i}$ and $U_{ \pm}$as complex over $\mathbb{C}_{j}$, the maps $\iota_{ \pm}: U_{0} \rightarrow U_{ \pm}$are complex-linear and conjugate-linear isomorphisms, 
respectively. If $U_{0}$ is an algebra, then $U$ is too, $U_{ \pm}$are ideals, and $\iota_{ \pm}: U_{0} \rightarrow U_{ \pm}$ are algebra isomorphisms.

If a real form of $U_{0}$ is specified, the associated $i$-conjugation $u \mapsto \bar{u}$ on $U_{0}$ extends to the $\mathbb{C}_{j}$-linear endomorphism of $U$ defined by $\iota_{ \pm}(u) \mapsto \iota_{\mp}(\bar{u})$.

Lemma 6.2. Suppose that $A$ and $B$ are vector spaces over $\mathbb{C}_{i}$ and that $B_{0}$ is a real form of $B$. If $L_{0}: A \rightarrow B_{0}$ is an $\mathbb{R}$-linear map, then there exists a unique $\mathbb{C}_{i}$-linear map $L: A \rightarrow B$ such that $L_{0}=\operatorname{Re}(L)$.

For the rest of this section, fix a complex Lie algebra $g_{0}$ over $\mathbb{C}_{i}$ and let $\mathrm{g}:=$ $\mathfrak{g}_{0} \otimes_{\mathbb{R}} \mathbb{C}_{j}$ be its complexification. Since $\mathfrak{g}$ is the direct sum of its ideals $\mathfrak{g}_{+}$and $\mathfrak{g}_{-}$,

$$
\mathfrak{U}(\mathfrak{g})=\mathfrak{U}\left(\mathfrak{g}_{+}\right) \otimes_{\mathbb{C}_{j}} \mathfrak{U}\left(\mathfrak{g}_{-}\right), \quad 3(\mathfrak{g})=3\left(\mathfrak{g}_{+}\right) \otimes_{\mathbb{C}_{j}} 3\left(\mathfrak{g}_{-}\right)
$$

as algebras, and $\iota_{ \pm}$extend to complex-linear and conjugate-linear isomorphisms from $\mathfrak{U}\left(\mathfrak{g}_{0}\right)$ (over $\left.\mathbb{C}_{i}\right)$ to $\mathfrak{U}\left(\mathfrak{g}_{ \pm}\right)$(over $\left.\mathbb{C}_{j}\right)$.

Let $V$ be a $\mathbb{C}_{i}$-space of real form $V_{0}$, and suppose that $\pi: \mathfrak{g}_{0} \rightarrow \operatorname{End}_{\mathbb{C}_{i}}(V)$ is an $\mathbb{R}$-linear representation. Then there are $\mathbb{R}$-linear maps $\operatorname{Re}(\pi)$ and $\operatorname{Im}(\pi)$ from $g_{0}$ to $\operatorname{End}_{\mathbb{R}}\left(V_{0}\right)$ such that $\pi=\operatorname{Re}(\pi)+i \operatorname{Im}(\pi)$, and we have the conjugate representation $\bar{\pi}:=\operatorname{Re}(\pi)-i \operatorname{Im}(\pi)$, another $\mathbb{R}$-linear representation of $g_{0}$ in $\operatorname{End}_{\mathbb{C}_{i}}(V)$.

Now apply Lemma 6.2 to $\frac{1}{2} \operatorname{Re}(\pi)$ and $\frac{1}{2} \operatorname{Im}(\pi)$ with $g_{0}$ for $A$ and $\operatorname{End}_{\mathbb{R}}\left(V_{0}\right)$ for $B_{0}$. This yields $\mathbb{C}_{i}$-linear maps $R_{\pi}, I_{\pi}: \mathfrak{g}_{0} \rightarrow \operatorname{End}_{\mathbb{C}_{i}}(V)$ such that $\pi=2\left(\operatorname{Re}\left(R_{\pi}\right)+\right.$ $\left.i \operatorname{Re}\left(I_{\pi}\right)\right)$. Let $\bar{R}_{\pi}$ and $\bar{I}_{\pi}$ be their conjugates with respect to $V_{0}$. Note that $R_{\bar{\pi}}=R_{\pi}$ and $I_{\bar{\pi}}=-I_{\pi}$.

Extend $\pi$ to a complex-linear representation of $\mathrm{g}\left(\right.$ over $\left.\mathbb{C}_{j}\right)$ on $V\left(\right.$ over $\left.\mathbb{C}_{i}\right)$.

Lemma 6.3. $\pi \circ \iota_{+}$is $R_{\pi}+i I_{\pi}$, and $\pi \circ \iota_{-}$is $\bar{R}_{\pi}+i \bar{I}_{\pi}$, which is $\overline{\bar{\pi} \circ \iota_{+}}$. They are $\mathbb{C}_{i}$-linear and $\mathbb{C}_{i}$ conjugate-linear representations of $\mathrm{g}_{0}$ on $V$, respectively.

6.2. The algebra $\mathbb{D}_{m}$. In this section we will prove Theorem 2.3 . Since $g_{0}^{J}(\mathbb{C})$ is a $\mathbb{C}_{i}$-Lie algebra, we take its complexification to be $g_{0}^{J}(\mathbb{C}) \otimes_{\mathbb{R}} \mathbb{C}_{j}$. Similarly, let $\mathfrak{f}^{J}(\mathbb{C})$ and $\mathfrak{m}^{J}(\mathbb{C})$ be the $\mathbb{C}_{j}$-complexifications of $\mathfrak{E}_{0}^{J}(\mathbb{C})$ and $\mathfrak{m}_{0}^{J}(\mathbb{C})$ (note that the latter are not $\mathbb{C}_{i}$-spaces).

Recall from Proposition 2.2 that conjugation by $y^{-k}$ is an equivalence from $\left.\right|_{0, m}$ to $\left.\right|_{k, m}$. Therefore it is also an isomorphism from the algebra of $\left.\right|_{0, m}$-invariant operators to that of $\left.\right|_{k, m}$-invariant operators, and so from now on it suffices to consider only the case $k=0$.

Let use the pattern of abbreviation established in Section 5.2, without the superscript $\mathbb{R}$ or the subscript $k$. Thus $\mathbb{D}_{m}$ is the algebra of $\left.\right|_{0, m}$-invariant differential operators, and $\lambda_{m}$ is the left action corresponding to $\left.\right|_{0, m}$. We have also the spaces $\mathcal{E}_{m}, \mathcal{I}_{m}$, and $\mathscr{D}_{m}$ from (34), the filtration-preserving algebra isomorphism $R_{m}: \mathscr{D}_{m} \rightarrow \mathbb{D}_{m}$ from (36), and the linear bijections from Section 4.3:

$$
\delta_{m}: S\left(\mathfrak{m}^{J}(\mathbb{C})\right)^{\mathfrak{k}^{J}(\mathbb{C})} \rightarrow \mathscr{D}_{m}, \quad \mathrm{IDO}_{m}=R_{m} \circ \delta_{m} \circ(-1)^{\text {degree }} .
$$




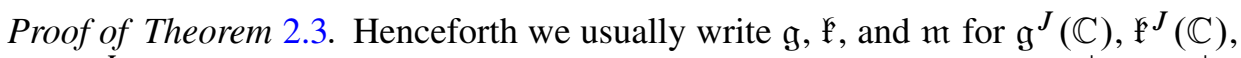
and $\mathfrak{m}^{J}(\mathbb{C})$, and for $\Omega \in \mathcal{U}(\mathfrak{g})$ we set $\Omega_{ \pm}:=\iota_{ \pm}(\Omega)$. First we prove that $\mathcal{C}_{m}^{ \pm}:=\mathcal{C}_{0, m}^{ \pm}$ are invariant and generate a polynomial algebra which is the image of $3(\mathrm{~g})$ under $\lambda_{m}$. Combine Proposition 5.2 and (46) to obtain:

Proposition 6.4. $3\left(\mathrm{~g}^{J}(\mathbb{C})\right)$ is the polynomial algebra $\mathbb{C}\left[Z_{+}, Z_{-}, C_{+}, C_{-}\right]$.

As a representation of $\mathfrak{g}_{0}, \lambda_{m}$ is $\mathbb{R}$-linear and acts by $\mathbb{C}_{i}$-linear endomorphisms on $C^{\infty}\left(\mathbb{H}_{Q} \times \mathcal{Q}\right)$, which has a natural real form: the real-valued functions. Thus we are in the setting of Lemma 6.3. The values of $\lambda_{m}$ on $\{B, i B: B=H, E, F, Z, e, f\}$ are given on page 153 of [4], except for the formula $\lambda_{m}(s Z)=-4 \pi i \operatorname{Re}\left({ }^{t} m m s\right)$. They are given in a form which makes it straightforward to deduce the $\mathbb{C}_{i}$-linear maps $R_{\lambda_{m}}$ and $I_{\lambda_{m}}$ of Lemma 6.3, giving the following lemma.

Lemma 6.5. For $B \in\{H, E, F, Z, e, f\}, \lambda_{m}\left(B_{+}\right)=B_{0, m}^{+}$(see (5)). The conjugate representation $\bar{\lambda}_{m}$ is $\lambda_{\text {im }}$, and so $\lambda_{m}\left(B_{-}\right)=\overline{\lambda_{\text {im }}\left(B_{+}\right)}=B_{0, m}^{-}$(see (6)).

Considering $\lambda_{m}$ as a complex-linear representation of $\mathfrak{g}$, it is now clear that $\lambda_{m}\left(Z_{ \pm}\right)$are scalars and $\lambda_{m}\left(C_{ \pm}\right)=-\pi i \ell_{m}^{ \pm}$. In light of Section $4.1, \ell_{m}^{ \pm}$generate $\lambda_{m}(3(\mathrm{~g}))$. To prove that they are algebraically independent, verify that their symbols are given by

$$
-\pi i \ell_{m}^{+} \equiv y^{2}\left(\partial_{\bar{x}} \partial_{u}^{2}-\partial_{x} \partial_{\bar{v}}^{2}+\partial_{y} \partial_{u} \partial_{\bar{v}}\right)+y v\left(\partial_{u} \partial_{\bar{u}}+\partial_{v} \partial_{\bar{v}}\right) \partial_{u}, \quad \ell_{m}^{-} \equiv \overline{\ell_{m}^{+}} .
$$

Suppose that they satisfy a degree $d$ relation. The symbol of this relation is of the form $\sum_{i=0}^{d} c_{i}\left(\mathcal{C}_{m}^{+}\right)^{d-i}\left(\mathcal{C}_{m}^{-}\right)^{i}$, the degree $3 d$ symbol of which is zero. Applying it to $x^{j} \bar{x}^{d-j} u^{2(d-j)} \bar{u}^{2 j}$ and evaluating at $(\mathbf{k}, 0)$ shows that $c_{i}=0$ for all $i$, so the relation is trivial.

It remains to prove that $\ell_{m}^{ \pm}$generate the center $Z\left(\mathbb{D}_{m}\right)$ of $\mathbb{D}_{m}$. Since $R_{m}: \mathscr{D}_{m} \rightarrow$ $\mathbb{D}_{m}$ is an algebra isomorphism, it suffices to prove that the natural images $C_{ \pm}+\mathcal{I}_{m}$ of $C_{ \pm}$in $\mathscr{D}_{m}$ generate $Z\left(\mathscr{D}_{m}\right)$. We will need a basis of $\mathscr{D}_{m}$, which we will obtain by constructing a basis of $S(\mathfrak{m})^{\mathfrak{E}}$ and applying (47). Verify that

$$
\begin{aligned}
\mathfrak{k} & =\operatorname{Span}_{\mathbb{C}_{j}}\left\{H_{+}-H_{-}, E_{+}-F_{-}, F_{+}-E_{-}, Z_{ \pm}\right\}, \\
\mathfrak{m} & =\operatorname{Span}_{\mathbb{C}_{j}}\left\{H_{+}+H_{-}, E_{+}+F_{-}, F_{+}+E_{-}, e_{ \pm}, f_{ \pm}\right\} .
\end{aligned}
$$

As a $\mathfrak{k}$-module, $\mathfrak{m}$ splits as $\mathfrak{m}_{2} \oplus \mathfrak{m}_{+} \oplus \mathfrak{m}_{-}$, where

$$
\mathfrak{m}_{2}:=\operatorname{Span}\left\{H_{+}+H_{-}, E_{+}+F_{-}, F_{+}+E_{-}\right\}, \quad \mathfrak{m}_{ \pm}:=\operatorname{Span}\left\{e_{ \pm}, f_{ \pm}\right\} .
$$

Recall from Section 5.1 the standard basis $\{H, E, F\}$ of $\mathfrak{s l}_{2}$, and its action on the module $L_{1}=\operatorname{Span}\{e, f\}$. It is easy to see that $H_{+}-H_{-}, E_{+}-F_{-}$, and $F_{+}-E_{-}$ are a standard basis of the copy of $\mathfrak{s I}_{2}$ in $\mathfrak{k}$, and that

$$
H_{+}-H_{-} \mapsto H_{+}+H_{-}, \quad E_{+}-F_{-} \mapsto E_{+}+F_{-}, \quad F_{+}-E_{-} \mapsto F_{+}+E_{-}
$$


defines a $\mathfrak{k}$-equivalence from $\mathfrak{k}$ to $\mathfrak{m}_{2}$. Also,

$$
e \mapsto e_{+}, \quad f \mapsto f_{+} ; \quad e \mapsto f_{-}, \quad f \mapsto-e_{-}
$$

define equivalences from the $\mathfrak{s l}_{2}$-action on $L_{1}$ to the $\mathfrak{k}$-actions on $\mathfrak{m}_{ \pm}$.

A little work with (44) and (45) gives the following lemma. Keeping in mind that in $\mathfrak{g}, X$ and $i X$ are independent over $\mathbb{C}_{j}$, define the following elements of $S(\mathfrak{m})$ :

$$
\begin{aligned}
Q_{\tau}:= & 4\left(F_{+}+E_{-}\right)\left(E_{+}+F_{-}\right)+\left(H_{+}+H_{-}\right)^{2} \\
= & (F+E)^{2}+(i F-i E)^{2}+H^{2}, \\
Q_{z}:= & 4\left(f_{+} f_{-}+e_{+} e_{-}\right)=f^{2}+(i f)^{2}+e^{2}+(i e)^{2}, \\
\Gamma_{0}:= & 4\left(2\left(F_{+}+E_{-}\right) e_{+} f_{-}+2\left(E_{+}+F_{-}\right) f_{+} e_{-}\right) \\
& \left.\quad+\left(H_{+}+H_{-}\right)\left(f_{+} f_{-}-e_{+} e_{-}\right)\right), \\
\Gamma_{+}:= & 8\left(\left(E_{+}+F_{-}\right) f_{+}^{2}-\left(F_{+}+E_{-}\right) e_{+}^{2}-\left(H_{+}+H_{-}\right) f_{+} e_{+}\right), \\
\Gamma_{-}:= & 8\left(\left(F_{+}+E_{-}\right) f_{-}^{2}-\left(E_{+}+F_{-}\right) e_{-}^{2}-\left(H_{+}+H_{-}\right) f_{-} e_{-}\right) .
\end{aligned}
$$

Lemma 6.6. $\delta^{2}(\mathfrak{m})^{\mathfrak{k}}=\operatorname{Span}\left\{Q_{\tau}, Q_{z}\right\}$, and $S^{3}(\mathfrak{m})^{\mathfrak{k}}=\operatorname{Span}\left\{\Gamma_{0}, \Gamma_{+}, \Gamma_{-}\right\}$.

This lemma matches Lemma 5.2 of [4]; the invariants $Q_{\tau}, Q_{z}, C_{0}, C_{+}$, and $C_{-}$given there are our $Q_{\tau}, Q_{z}, \Gamma_{0}, j \Gamma_{+}$, and $j \Gamma_{-}$, respectively. Note that the $i$ conjugation defined by the usual real form of $\mathrm{g}_{0}$ fixes $Q_{\tau}, Q_{z}$, and $\Gamma_{0}$ and exchanges $\Gamma_{+}$and $\Gamma_{-}$.

In Theorem 5.3 of [4] we proved that these five invariants generate $S(\mathfrak{m})^{\mathfrak{k}}$ and have a relation ideal generated by a single relation. However, only a sketch of the argument was given; here we shall give a complete proof.

Proposition 6.7. The set $\left\{Q_{\tau}^{i_{\tau}} \Gamma_{0}^{i_{0}} Q_{z}^{i_{z}} \Gamma_{+}^{i_{+}} \Gamma_{-}^{i_{-}}: i_{\tau}, i_{z}, i_{p}, i_{m} \in \mathbb{N}, i_{0}=0,1\right\}$ is a basis of $S(\mathfrak{m})^{\mathfrak{k}}$, and

$$
\Gamma_{0}^{2}+\Gamma_{+} \Gamma_{-}-Q_{\tau} Q_{z}^{2}=0 .
$$

Proof. To prove (48), write $\oint^{i, j, k}(\mathfrak{m})$ for the $\mathfrak{k}^{\mathfrak{s}-\text { submodule }} \oint^{i}\left(\mathfrak{m}_{2}\right) \mathcal{S}^{j}\left(\mathfrak{m}_{+}\right) \mathcal{S}^{k}\left(\mathfrak{m}_{-}\right)$ of $\delta(\mathfrak{m})$. By (44), $\delta^{2,2,2}(\mathfrak{m})$ is $\mathfrak{k}$-equivalent to $\left(L_{4} \oplus L_{0}\right) \otimes L_{2} \otimes L_{2}$, which has a 2-dimensional space of invariants. Hence $\Gamma_{0}^{2}, \Gamma_{+} \Gamma_{-}$, and $Q_{\tau} Q_{z}^{2}$ must be related. Keeping track of only certain terms (for example the coefficients of $e^{4}$ or of $H^{2}$ ) gives (48) quickly.

Write $\mathscr{B}$ for our proposed basis. In light of (48), proving that $S(\mathfrak{m})^{\mathfrak{k}}$ equals Span $\mathscr{B}$ is equivalent to proving that our five invariants generate it.

Write $H_{\mathfrak{F}}, E_{\mathfrak{k}}$, and $F_{\mathfrak{F}}$ for the basis elements $H_{+}-H_{-}, E_{+}-F_{-}$, and $F_{+}-E_{-}$ of the copy of $\mathfrak{s l}_{2}$ in $\mathfrak{k}$, and $H_{\mathfrak{m}}, E_{\mathfrak{m}}$, and $F_{\mathfrak{m}}$ for the basis elements $H_{+}+H_{-}$, 
$E_{+}+F_{-}$, and $F_{+}+E_{-}$of $\mathfrak{m}_{2}$. Suppose that $P$ is an invariant of degree $r$ in $E_{\mathfrak{m}}$. Assume by induction that invariants of lower $E_{\mathfrak{m}}$-degree are in Span $\mathcal{B}$. We may assume further that $P$ is homogeneous of degree $r+s$ in $\mathfrak{m}_{2}$, so the coefficient of $E_{\mathfrak{m}}^{r}$ in $P$ is $\sum_{i=0}^{s} H_{\mathfrak{m}}^{s-i} F_{\mathfrak{m}}^{i} p_{i}$ for unique $p_{i} \in \mathbb{C}\left[e_{ \pm}, f_{ \pm}\right]$.

Considering $\operatorname{ad}\left(E_{\mathfrak{k}}\right) P=0$, one finds that $p_{i}=0$ for $i<s$. Then considering $\operatorname{ad}\left(F_{\mathfrak{E}}\right) P=0$ leads to $\operatorname{ad}\left(F_{\mathfrak{F}}\right) p_{s}=0$. Thus modulo terms of lower $E_{\mathfrak{m}}$-degree, $P \equiv E_{\mathfrak{m}}^{r} F_{\mathfrak{m}}^{s} p_{s}$, where $p_{s}$ is a $\mathfrak{k}^{-}$-lowest weight vector of $\delta\left(\mathfrak{m}_{+} \oplus \mathfrak{m}_{-}\right)$.

Using (44), it is not hard to prove that

$$
S\left(\mathfrak{m}_{+} \oplus \mathfrak{m}_{-}\right)=\mathbb{C}\left[Q_{z}\right] \otimes \bigoplus_{a, b \in \mathbb{N}} L\left(\mathfrak{m}_{+} \oplus \mathfrak{m}_{-}, a, b\right),
$$

where $L\left(\mathfrak{m}_{+} \oplus \mathfrak{m}_{-}, a, b\right)$ is the $\mathfrak{k}$-copy of $L_{a+b}$ of highest weight vector $e_{+}^{a} f_{-}^{b}$ and lowest weight vector $f_{+}^{a} e_{-}^{b}$. By (49), $p_{s} \in \operatorname{Span}_{\mathbb{C}\left[Q_{z}\right]}\left\{f_{+}^{a} e_{-}^{b}: a+b=r-s\right\}$. Therefore we can lower $P$ 's $E_{\mathfrak{m}}$-degree by subtracting an element of Span $\mathscr{B}$, proving Span $\mathcal{B}=S(\mathfrak{m})^{\mathfrak{k}}$.

To prove that the elements of $\mathscr{B}$ are independent, use (44) again to prove that

$$
\delta\left(\mathfrak{m}_{2}\right)=\mathbb{C}\left[Q_{\tau}\right] \otimes \bigoplus_{c \in 2 \mathbb{N}} L\left(\mathfrak{m}_{2}, c\right),
$$

where $L\left(\mathfrak{m}_{2}, c\right)$ is the $\mathfrak{k}$-copy of $L_{c}$ of highest weight vector $E_{\mathfrak{m}}^{c / 2}$ and lowest weight vector $F_{\mathfrak{m}}^{c / 2}$ (this is Kostant's harmonic factorization for $\left.\mathfrak{s}_{2}\right)$. For $a+b$ even, let $P(a, b)$ be the unique invariant in $L\left(\mathfrak{m}_{2}, a+b\right) \otimes L\left(\mathfrak{m}_{+} \oplus \mathfrak{m}_{-}, a, b\right)$. By (49) and (50), the $P(a, b)$ are independent over $\mathbb{C}\left[Q_{\tau}, Q_{z}\right]$. Examining the proof of Span $\mathcal{B}=S(\mathfrak{m})^{\mathfrak{k}}$ and using (48), one finds that $\{P(a+b): a+b \leq 2 N\}$ and $\left\{\Gamma_{0}^{i_{0}} \Gamma_{+}^{i_{+}} \Gamma_{-}^{i_{-}}: i_{0}+i_{p}+i_{m} \leq N, i_{0}=0,1\right\}$ have the same span over $\mathbb{C}\left[Q_{\tau}, Q_{z}\right]$ and the same cardinality. The result follows.

We now prove that $C_{ \pm}+\mathcal{I}_{m}$ generate $Z\left(\mathscr{D}_{m}\right)$. Define elements $\delta_{\bullet}(\mathbb{C}, m)$ (we usually write simply $\delta_{\bullet}$ ) of $\mathscr{D}_{m}$ as follows:

$\delta_{z}:=\delta_{m}\left(Q_{z}\right), \quad \delta_{\tau}:=\delta_{m}\left(Q_{\tau}\right), \quad \delta_{0}:=\delta_{m}\left(\Gamma_{0}\right), \quad \delta_{+}:=\delta_{m}\left(\Gamma_{+}\right), \quad \delta_{-}:=\delta_{m}\left(\Gamma_{-}\right)$.

By Proposition 6.7, $\left\{\delta_{\tau}^{i_{\tau}} \delta_{0}^{i_{0}} \delta_{z}^{i_{z}} \delta_{+}^{i_{+}} \delta_{-}^{i_{-}}: i_{\tau}, i_{z}, i_{p}, i_{m} \in \mathbb{N}, i_{0}=0,1\right\}$ is a basis of $\mathscr{D}_{m}$. Using the following lemma, a calculation at the symbol level exactly analogous to the one at the end of Section 5.2 gives the result.

Lemma 6.8. Up to symbols in $\mathscr{D}_{m}$,

$$
\delta_{ \pm} \equiv 16 C_{ \pm}, \quad \delta_{0}^{2} \equiv \delta_{\tau} \delta_{z}^{2}-\delta_{+} \delta_{-}, \quad\left[\delta_{z}, \delta_{\tau}\right] \equiv 4 \delta_{0}, \quad\left[\delta_{z}, \delta_{0}\right] \equiv 2 \delta_{z}^{2} .
$$

Proof. Let us review computation in $\mathscr{D}_{m}$. Let $\pi_{m}$ be the character of $K^{J}(\mathbb{C})$ associated to $\left.\right|_{0, m}$ by Section 4.2. (In fact, $\pi_{m}$ is the character associated to $\left.\right|_{k, m}$ for all $k$, 
and it sends $Z_{+}$to $-2 \pi i^{t} m m, Z_{-}$to $-2 \pi i^{t} \bar{m} \bar{m}$, and $H_{\mathfrak{F}}, E_{\mathfrak{k}}$, and $F_{\mathfrak{k}}$ to zero, but we shall not need this.) Here $\mathcal{E}_{m}=\mathfrak{U}(\mathfrak{g})=\operatorname{Sym}(S(\mathfrak{m})) \cdot \mathfrak{U}(\mathfrak{k})$. Consider a pure product $\Omega=\operatorname{Sym}\left(\Omega_{\mathfrak{m}}\right) \Omega_{\mathfrak{k}}$ in this space. By (35), it is congruent to $\operatorname{Sym}\left(\Omega_{\mathfrak{m}}\right) \pi_{m}\left(\Omega_{\mathfrak{F}}^{T}\right)$. In particular, if $\Omega_{\mathfrak{k}}$ has non-zero constant term $\omega$, then $\Omega$ and $\omega \Omega_{\mathfrak{m}}$ have the same degree and symbol modulo $\tilde{I}_{m}$.

Since $\Gamma_{ \pm}$are cubic in $\mathfrak{m}$, the symbols of $\delta_{ \pm}$modulo $\mathcal{I}_{m}$ are independent of $m$ and may be computed by setting all terms in $\Gamma_{ \pm}$from $\mathfrak{f}^{\mathrm{k}}$ to zero, i.e., setting $Z_{ \pm} \equiv 0$, $H_{+} \equiv H_{-}, E_{+} \equiv F_{-}$, and $F_{+} \equiv E_{-}$. Checking Definition 5 gives the first congruence. The second is immediate from (48) and the fact that $\delta_{m}$ is an algebra isomorphism up to symbol.

For the last two congruences, note that for any $\Theta$ and $\Theta^{\prime}$ in $\mathcal{U}(\mathfrak{g})$, the symbol of $\left[\Theta, \Theta^{\prime}\right]$ depends only on the symbols of $\Theta$ and $\Theta^{\prime}$. It follows that we need only compute the symbols of $\left[Q_{z}, Q_{\tau}\right]$ and $\left[Q_{z}, \Gamma_{0}\right]$, treating $Q_{z}, Q_{\tau}$, and $\Gamma_{0}$ as elements of $\mathcal{E}_{m} / \mathcal{I}_{m}$. Commutators of elements of $\mathfrak{m}$ which lie in $\mathfrak{k}$ give zero modulo symbol, so we only keep commutators of $H_{\mathfrak{m}}, E_{\mathfrak{m}}$, and $F_{\mathfrak{m}}$ with $e_{ \pm}$and $f_{ \pm}$. Therefore up to symbol, $\left[Q_{z}, Q_{\tau}\right]$ must be of degree $(1,1,1)$ and $\left[Q_{z}, \Gamma_{0}\right]$ must be of degree $(0,2,2)$. It only remains to compute the constants of proportionality, for which one need only keep track of one term.

6.3. Remarks. We leave the reader with some open problems. First, the irreducible representations of $K^{J}(\mathbb{C})=\mathrm{SU}_{2} \times \mathbb{R}^{2}$ are parametrized by $\left(n, m, m^{\prime}\right) \in \mathbb{N} \times \mathbb{C} \times \mathbb{C}$, where $n+1$ is the dimension. As we mentioned in [4], a study of the invariant differential operators of the associated vector-valued slash actions would be a natural extension of both our work and that of Friedberg [9]. Equation (36) would be useful.

Second, the characters of the algebras $\mathbb{D}_{m}$ and $\mathbb{D}_{k, m}^{\mathbb{R}}$ may be of interest, as these algebras must act by scalars on any slash-irreducible subrepresentation of the space of functions on which they act. These characters are of course simply the characters of the abelianizations $\mathbb{D}_{m} /\left[\mathbb{D}_{m}, \mathbb{D}_{m}\right]$ and $\mathbb{D}_{k, m}^{\mathbb{R}} /\left[\mathbb{D}_{k, m}^{\mathbb{R}}, \mathbb{D}_{k, m}^{\mathbb{R}}\right]$, and it appears to be reasonably easy to compute all of them.

Finally, regarding $\mathbb{D}_{m}$ as a deformation of $\mathbb{D}_{0}$ yields an unobstructed 2-class in the Hochschild cohomology of $\mathbb{D}_{m}$. Similarly, $\mathbb{D}_{k, m}^{\mathbb{R}}$ gives two 2-classes in the cohomology of $\mathbb{D}_{0,0}^{\mathbb{R}}$. It could be amusing to compute these cohomology spaces and decide whether or not the 2-classes are trivial. Theorem 5.3 would be useful in the real case; one would need its complex analog for the complex case. The computations might be reduced by using the $\mathbb{C}_{i}$-linear automorphisms $\theta$ and $\tau$ of $\mathfrak{g}_{0}$ defined in Section 5.1. They both preserve $\mathfrak{f}_{0}$ and $\mathfrak{m}_{0}$, and so they extend to $\mathbb{C}_{j}$-linear automorphisms of $\mathfrak{g}$ which commute with $\iota_{ \pm}$and preserve $\mathfrak{k}$ and $\mathfrak{m}$.

Acknowledgments. We thank Ameya Pitale and Ralf Schmidt for interesting discussions which led to this paper, and the referee for thoughtful comments which improved it. 


\section{References}

[1] R. Berndt and R. Schmidt, Elements of the representation theory of the Jacobi group. Progr. Math. 163, Birkhäuser, Basel 1998. Zbl 0931.11013 MR 1634977

[2] A. Borel, Introduction to automorphic forms. In Algebraic groups and discontinuous subgroups, (Proc. Sympos. Pure Math., Boulder, Colo., 1965), Amer. Math. Soc., Providence, R.I., 1966, 199-210. Zbl 0195.09501 MR 0207650

[3] W. Borho, Primitive und vollprimitive Ideale in Einhüllenden von $\mathfrak{s}(5, \mathbb{C})$. J. Algebra 43 (1976), 619-654. Zbl 0346.17013 MR 0422368

[4] K. Bringmann, C. Conley and O. Richter, Maass-Jacobi forms over complex quadratic fields. Math. Res. Lett. 14 (2007), no. 1, 137-156. Zbl 1132.11024 MR 2289627

[5] J. Cardy, Operator content of two-dimensional conformally invariant theories. Nuclear Phys. B 270 (1986), no. 2, 186-204. Zbl 0689.17016 MR 0845940

[6] Y. Choie and O. Richter, Classification of the space spanned by theta series and applications. Proc. Amer. Math. Soc. 135 (2007), no. 8, 2309-2315. Zbl 1165.11044 MR 2302551

[7] M. Eichler and D. Zagier, The theory of Jacobi forms. Progr. Math. 55, Birkhäuser, Boston, 1985. Zbl 0554.10018 MR 0781735

[8] J. Elstrodt, F. Grunewald and J. Mennicke, Groups acting on hyperbolic space. Springer Monogr. Math., Springer-Verlag, Berlin 1998. Zbl 0888.11001 MR 1483315

[9] S. Friedberg, Differential operators and theta series. Trans. Amer. Math. Soc. 287 (1985), no. 2, 569-589. Zbl 0524.10020 MR 0768726

[10] B. Gross, W. Kohnen and D. Zagier, Heegner points and derivatives of $L$-series. II. Math. Ann. 278 (1987), no. 1-4, 497-562. Zbl 0641.14013 MR 0909238

[11] S. Helgason, Invariant differential equations on homogeneous manifolds. Bull. Amer. Math. Soc. 84 (1977), no. 5, 751-774. Zbl 0377.43009 MR 0445235

[12] S. Helgason, Some results on invariant differential operators on symmetric spaces. Amer. J. Math. 114 (1992), no. 4, 789-811. Zbl 0838.43012 MR 1175692

[13] Ö. Imamoḡlu, The Kubota symbol for $\operatorname{Sp}(2 n, Q(i))$. J. Number Theory 52 (1995), 17-34. Zbl 0834.11022 MR 1331762

[14] W. Kohnen, Modular forms of half-integral weight on $\Gamma_{0}$ (4). Math. Ann. 248 (1980), no. 3 , 249-266. Zbl 0416.10023 MR 0575942

[15] H. Kojima, On the Fourier coefficients of Maass wave forms of half integral weight over an imaginary quadratic field. J. Reine Angew. Math. 526 (2000), 155-179. Zbl 0987.11033 MR 1778303

[16] J. Mickelsson, Step algebras of semi-simple subalgebras of Lie algebras. Rep. Math. Phys. 4 (1973), 307-318. Zbl 0285.17005 MR 0342057

[17] A. Pitale, Jacobi Maaß forms. Abh. Math. Sem. Univ. Hamburg 79 (2009), 87-111. Zbl 05620554 MR 2541345

[18] O. Richter and H. Skogman, Jacobi theta functions over number fields. Monatsh. Math. 141 (2004), no. 3, 219-235. Zbl 0956.11013 MR 1739742

[19] O. Richter, Theta functions of quadratic forms over imaginary quadratic fields. Acta Arith. 92 (2000), no. 1, 1-9. Zbl 1111.11026 MR 2042213 
[20] H. Skogman, Jacobi forms over imaginary quadratic number fields. Acta Arith. 96 (2001), no. 4, 333-350. Zbl 1077.11035 MR 1811875

[21] N.-P. Skoruppa, Developments in the theory of Jacobi forms. In Automorphic functions and their applications, Acad. Sci. USSR, Inst. Appl. Math., Khabarovsk 1990, 167-185. Zbl 0745.11029 MR 1096975

[22] N.-P. Skoruppa, Holomorphe und schiefholomorphe Jacobiformen. In preparation.

[23] N.-P. Skoruppa and D. Zagier, Jacobi forms and a certain space of modular forms. Invent. Math. 94 (1988), no. 1, 113-146. Zbl 0651.10020 MR 0958592

[24] D. Zagier, Note on the Landweber-Stong elliptic genus. In Elliptic curves and modular forms in algebraic topology (Princeton, NJ, 1986), Lecture Notes in Math. 1326, SpringerVerlag, Berlin 1988, 216-224. Zbl 0653.57016 MR 0970290

[25] C. Ziegler, Jacobi forms of higher degree. Abh. Math. Sem. Univ. Hamburg, 59 (1989), 191-224. Zbl 0707.11035 MR 1049896

[26] S. Zwegers, Mock theta functions. Ph.D. thesis, Universiteit Utrecht, The Netherlands, 2002 .

Received October 12, 2009

Kathrin Bringmann, Mathematisches Institut, Universität Köln, Weyertal 86-90, 50931 Köln, Germany

E-mail: kbringma@math.uni-koeln.de

Charles H. Conley, Department of Mathematics, University of North Texas, Denton, TX 76203, U.S.A.

E-mail: conley@unt.edu

Olav K. Richter, Department of Mathematics, University of North Texas, Denton, TX 76203 , U.S.A.

E-mail: richter@unt.edu 